\title{
Maximizing dissipation in a turbulent shear flow by optimal control of its initial state
}

\author{
S. Delport, M. Baelmans, and J. Meyers ${ }^{\mathrm{a})}$ \\ Department of Mechanical Engineering, Katholieke Universiteit Leuven, Celestijnenlaan 300A-bus 2421, \\ B3001 Leuven, Belgium
}

(Received 14 September 2010; accepted 22 March 2011; published online 19 April 2011)

\begin{abstract}
Turbulent shear flows are known to evolve self-similar at sufficiently high Reynolds numbers. In this regime, flow properties remain constant when normalized with local large-scale reference values. Dissipation of turbulent energy occurs at small scales. Thus, controls which increase dissipation levels in a shear flow may lose their effectiveness once the shear flow attains self-similar behavior governed by large-scale motion. In the current work, we investigate the optimal control of kinetic-energy dissipation in a temporally evolving turbulent mixing layer, with controls acting on its initial condition. Gradient-based optimization is used, relying on direct numerical simulations, and adjoint formulations for the determination of the gradients. Focus is on long optimization time windows $T$ up to 160 nondimensional time units. First we investigate the optimal controls which maximize the total energy dissipation over a simulation time window. For increasing $T$, optimal controls are found to become also optimal for preceding time horizons. Results indicate that these controls facilitate a fast transition of the mixing layer to a self-similar state, which occurs at nondimensional time $t \approx 160-200$. We find that the transition Reynolds number at which self-similarity emerges is a factor of 4-5 lower than transition Reynolds numbers reported for uncontrolled temporal mixing layers. Second, we investigate the maximization of the rate of dissipation at the end of the simulation time window. It is observed that dissipation rate can be increased up to two times higher than self-similar dissipation levels. However, at long optimization time windows this leads to a delay of transition to self-similarity. We further find that optimization is strongly nonconvex, and for the second case, we identify two local optima, which are further discussed in terms of differences in coherent structures emerging during the mixing-layer evolution. (C) 2011 American Institute of Physics. [doi:10.1063/1.3579487]
\end{abstract}

\section{INTRODUCTION}

Free shear flows appear in many industrial and environmental applications. Controlling the dissipation in such systems can be important to, e.g., smear out velocity differences, increase mixing, control reaction rates in reacting shear flows, reduce noise production, etc. For sufficiently high Reynolds numbers, free shear flows attain a self-similar state, ${ }^{1-4}$ characterized by constant profiles in time or space for mean velocity, Reynolds stresses, production and dissipation of turbulent kinetic energy, etc., when appropriately normalized with the large-scale flow properties, such as, e.g., the shear-layer thickness. Dissipation is a small-scale turbulent phenomenon, but in case of high Reynolds number selfsimilar shear flows, it is governed by the large-scale production of turbulent kinetic energy. The turbulent small-scale dissipation range is in that case in quasi-equilibrium. Therefore, flow control which increases dissipation levels in a shear flow may lose its effectiveness once the shear flow attains self-similar behavior governed by large-scale motion. In the current work, we investigate the optimal control of dissipation in turbulent shear flows and the transition to selfsimilarity based on direct numerical simulations. For reasons

\footnotetext{
${ }^{a}$ Author to whom correspondence should be addressed. Electronic mail: johan.meyers@mech.kuleuven.be.
}

of computational resources, we focus on the temporal mixing layer. As a consequence of this choice, the "controls" in the current work correspond to optimal perturbations of the mixing layer's initial condition (as further detailed below). Hence, the optimal control problem considered in the current study differs from traditional open-loop or feed-back systems using control laws in time.

Growing computational resources and the development of massively parallel computer platforms have allowed for the combination of optimal control with direct numerical simulations (DNS) in recent years. Pioneering work, combining the theory of optimal control of partial differential equations, with transient three-dimensional Navier-Stokes simulations in the turbulent regime (i.e., using DNS), was performed in the context of drag reduction of turbulent boundary layers by Bewley et al. ${ }^{5}$ Lately, a lot of efforts have focused on the area of drag reduction, ${ }^{6}$ or bluff bodies, ${ }^{7}$ and the reduction of flow noise. ${ }^{8}$ In the studies of Bewley $e t$ $a l .{ }^{5}$ and Wei et al., ${ }^{8}$ controls are optimized using gradientbased algorithms in combination with adjoint-based determination of the gradient of the optimization cost functional. In the current work, we rely on a similar approach, tailored for a temporal mixing layer, and the optimal control of its initial condition.

Since the pioneering work of Brown and Roshko, ${ }^{9}$ the mixing layer has been studied intensively, and the mecha- 
nisms responsible for its transition to turbulence are well documented nowadays. In the 1990s, direct numerical simulations of the temporal mixing layer became feasible, and many simulation studies illustrated the roll-up of the KelvinHelmholtz instability, and the subsequent pairing and break-up processes which lead to a fully turbulent flow. ${ }^{3,4,10-13}$ The temporal framework was particularly attractive for its geometrical simplicity, allowing the use of efficient Fourier discretization, etc., contributing to the feasibility of these simulations on the computer systems of those days. In the current work, mixing-layer optimization requires $\mathcal{O}\left(10^{3}\right)$ flow simulations (including adjoint-based gradient estimations), dictating our choice for the temporal framework in view of current-day high-performance systems.

We consider the optimal control of the initial condition of a temporal mixing layer, i.e., the initial condition is constructed as a combination of a hyperbolic-tangent streamwise velocity profile, and a set of spatially distributed perturbations with zero mean, which correspond to our controls. The controls in this temporal framework could be imagined to be connected to a set of spatially distributed actuators on an upstream splitter plate in a corresponding spatial framework. However, in the current work, it is not our intention to elaborate a rigorous connection between the optimized temporal results and a mixing layer in a spatial framework. Instead, we focus on the optimal control of dissipation in the mixing layer's transition and its effects on the ensuing selfsimilar region.

The optimization of the initial condition of temporal mixing layers has been studied extensively based on the linearized Navier-Stokes equations, mainly focusing on modal (eigenvalue) analysis ${ }^{14-17}$ (the use of nonmodal stability theory has gained a lot of attention in more recent years, 18,19 but to our best knowledge, has not been used to study the temporal mixing layer). These studies mainly focus on the early growth rate of perturbations on an initial mean-flow condition and their further transition to turbulence. The current work focuses on optimization of the initial condition based on the nonlinear Navier-Stokes equations, with optimization cost functionals which strongly depend on the nonlinear flow evolution at longer time horizons. The optimization is performed to simulation time horizons well into the turbulent range with $\mathrm{Re}_{\lambda} \approx 40$.

The first case that is considered (case 1) is the maximization of the total kinetic energy dissipated over a simulation time window $[0, T]$. Different time horizons $T$ are considered, up to $T=160$ nondimensional time units, and two different energy levels for the controls are included. For increasing $T$, optimal controls are found to become also optimal for preceding time horizons. Results indicate that these controls facilitate a fast transition of the mixing layer to a self-similar state, which occurs at nondimensional time $t \approx 160-200$, and conclusions are independent of the energy levels used for the controls. In all these cases, we find that the maximum dissipation rate appears well before the onset of self-similarity during the early transition of the mixing layer (around $t=70$ ).

Therefore, we attempt in the second case (case 2) to maximize the dissipation rate at the time horizon $T$ for time horizons up to $T=160$. For this case, it is observed that dissipation levels can be controlled up to levels which are a factor of 2.5 higher than dissipation levels in the self-similar region. For long optimization time windows this leads to a delay of transition to self-similarity.

We found the optimization of controls in the temporal mixing layer to be strongly nonconvex, such that local optima may exist. In earlier work focusing on mixing-layer transition for low time horizons $(T \leq 40)$, it was observed that this can lead to small inconsistencies between optima obtained for different time horizons: ${ }^{20}$ in some cases, optimal perturbations for $T_{1}<T_{2}$ are found to lead to lower cost functionals at $t=T_{2}$ than perturbations optimized for $T_{2}$. To avoid this type of situations, we slightly adapt the optimization procedure in the current work using a simple continuation method in the space of optimal solutions. Nevertheless, for the second optimization case (maximizing dissipation rate), two strongly distinct local optima are found, depending on the starting point of the procedure. Differences between these optima are discussed, and coherent structures emerging during the mixing-layer evolution are shown.

The paper is further organized as follows. First, in Sec. II, the optimization problem, optimization technique, and computational setup are described. The optimization results are discussed in Secs. III and IV for case 1 and case 2, respectively. Conclusions are presented in Sec. V.

\section{OPTIMIZATION CASE AND COMPUTATIONAL SETUP}

\section{A. Optimization problem}

We optimize a temporal turbulent mixing layer by searching for an optimal set of initial perturbations which are imposed on a hyperbolic-tangent stream-wise mean velocity profile. In the current section, this optimization problem is defined in terms of cost functionals and constraints.

Conventionally, an optimization problem is formulated in terms of the minimization of a cost functional $\mathcal{J}$. We consider cost functionals $\mathcal{J}$ of the form

$$
\mathcal{J}[\boldsymbol{q}(\boldsymbol{\phi})]=\int_{\Omega} J[\boldsymbol{q}(\boldsymbol{x}, T, \boldsymbol{\phi})] \mathrm{d} \boldsymbol{x},
$$

with $\boldsymbol{q}(\boldsymbol{x}, T, \boldsymbol{\phi})$ a turbulent flow solution $\boldsymbol{q}=[\boldsymbol{u}, p]$ at time horizon $T$ and defined on the spatial domain $\Omega$, with $\boldsymbol{u}$ the velocity field, and $p$ the pressure. The flow solution $\boldsymbol{q}(\boldsymbol{x}, T, \boldsymbol{\phi})$ follows from the evolution of the mixing layer with initial condition

$$
\boldsymbol{u}(\boldsymbol{x}, 0, \boldsymbol{\phi})=\tanh \left(x_{3}\right) \mathbf{e}_{1}+\boldsymbol{\phi}(\boldsymbol{x}),
$$

with $\boldsymbol{\phi}(\boldsymbol{x})$ the optimization controls (i.e., the initial perturbations of the mixing layer) and $\mathbf{e}_{1}$ the unit vector in the stream-wise direction $x_{1}$.

The optimization problem is conventionally formulated as minimization

$$
\min _{\boldsymbol{\phi} \in L^{2}(\Omega)} \mathcal{J}[\boldsymbol{q}(\boldsymbol{\phi})],
$$

further subject to three additional constraints. First of all, the problem is a partial differential equation (PDE)-constrained 
optimization problem, since the evolution of the flow $\boldsymbol{q}(\boldsymbol{x}, t, \boldsymbol{\phi})$ is governed by the incompressible Navier-Stokes equations,

$$
\begin{aligned}
& \nabla \cdot \boldsymbol{u}=0, \\
& \frac{\partial \boldsymbol{u}}{\partial t}+\boldsymbol{u} \cdot \nabla \boldsymbol{u}+\nabla p-\frac{1}{\operatorname{Re}} \nabla^{2} \boldsymbol{u}=0,
\end{aligned}
$$

with Re the Reynolds number, and with initial conditions at $t=0$ given by Eq. (2). The controls $\boldsymbol{\phi}$ are subject to two further constraints, i.e.,

$$
\begin{aligned}
& \nabla \cdot \boldsymbol{\phi}(\boldsymbol{x})=0, \\
& \frac{1}{2} \frac{1}{\Omega} \int_{\Omega} \boldsymbol{\phi} \cdot \boldsymbol{\phi} \mathrm{d} \boldsymbol{x}=E_{0} \frac{(\Delta U)^{2}}{8} .
\end{aligned}
$$

Constraint [Eq. (6)] corresponds with the requirement that the initial velocity field $\boldsymbol{u}(\boldsymbol{x}, 0, \boldsymbol{\phi})$ satisfies continuity [cf. Eqs. (2) and (4)]. Further, Eq. (7) keeps the total energy per unit volume of the perturbations fixed at a predefined constant level $E_{0}(\Delta U)^{2} / 8$, where the energy of the perturbations $E_{0}$ is expressed relative to the mean-flow kinetic energy per volume $(\Delta U)^{2} / 8$ (for an infinite box; with $\Delta U=2$ being the velocity difference between the upper and the lower streams of the mixing layer).

As discussed in Sec. I, we focus on two cost functionals in the current study. The first cost functional maximizes the total dissipated kinetic energy up to the time horizon. Since the volume-averaged dissipation $\varepsilon=2 \nu \mathcal{E}$, with the volumeaveraged enstrophy defined as

$$
\mathcal{E}(T, \boldsymbol{\phi})=\frac{1}{2 \Omega} \int_{\Omega} \boldsymbol{\omega}(\boldsymbol{x}, T, \boldsymbol{\phi}) \cdot \boldsymbol{\omega}(\boldsymbol{x}, T, \boldsymbol{\phi}) \mathrm{d} \boldsymbol{x},
$$

and $\omega=\nabla \times \boldsymbol{u}$, we can now define a cost functional which maximizes the volume-averaged dissipation of energy over a time window $[0, T]$ as

$$
\begin{aligned}
\mathcal{J}_{\mathrm{DE}}= & -\frac{\nu}{\Omega} \int_{0}^{T} \int_{\Omega} \boldsymbol{\omega}(\boldsymbol{x}, t, \boldsymbol{\phi}) \cdot \boldsymbol{\omega}(\boldsymbol{x}, t, \boldsymbol{\phi}) \mathrm{d} \boldsymbol{x} \mathrm{d} t, \\
= & -\left[\left(1+E_{0}\right)(\Delta U)^{2} / 8\right. \\
& \left.-\frac{1}{2} \frac{1}{\Omega} \int_{\Omega} \boldsymbol{u}(\boldsymbol{x}, T, \boldsymbol{\phi}) \cdot \boldsymbol{u}(\boldsymbol{x}, T, \boldsymbol{\phi}) \mathrm{d} \boldsymbol{x}\right] .
\end{aligned}
$$

In Eq. (10) the total dissipated energy is expressed as the difference between the total energy at $t=0$ and the energy at $t=T$, and we use this formulation of the cost functional in the optimization procedure.

A cost functional that maximizes the dissipation rate at the time horizon $T$ is straightforwardly expressed as

$$
\mathcal{J}_{\mathrm{DR}}=-\frac{\nu}{\Omega} \int_{\Omega} \boldsymbol{\omega}(\boldsymbol{x}, T, \boldsymbol{\phi}) \cdot \boldsymbol{\omega}(\boldsymbol{x}, T, \boldsymbol{\phi}) \mathrm{d} \boldsymbol{x} .
$$

For the sake of convenience, we will denote optimal perturbations for a time horizon $T$ using $\boldsymbol{\phi}_{T}$ (e.g., for $T=60$ we write $\left.\boldsymbol{\phi}_{60}\right)$. We will evaluate the optimal perturbations by looking at the mixing layer's evolution of total dissipated energy in time defined as

$$
\Delta E\left(t, \boldsymbol{\phi}_{T}\right)=\frac{\nu}{\Omega} \int_{0}^{t} \int_{\Omega} \boldsymbol{\omega}\left(\boldsymbol{x}, t^{\prime}, \boldsymbol{\phi}\right) \cdot \boldsymbol{\omega}\left(\boldsymbol{x}, t^{\prime}, \boldsymbol{\phi}\right) \mathrm{d} \boldsymbol{x} \mathrm{d} t^{\prime} .
$$

Likewise, we will monitor the dissipation rate by evaluating the volume-averaged enstrophy in time, i.e., $\mathcal{E}\left(t, \boldsymbol{\phi}_{T}\right)$, using its definition [Eq. (8)]. It is clear that these properties are related to the optimization cost functionals as $\Delta E\left(T, \boldsymbol{\phi}_{T}\right)$ $=-\mathcal{J}_{\mathrm{DE}}$ and $\mathcal{E}\left(T, \boldsymbol{\phi}_{T}\right)=-\mathcal{J}_{\mathrm{DR}} /(2 \nu)$.

\section{B. Optimization technique}

A gradient-based optimization algorithm is employed for the optimization, similar to the techniques used in the work of, e.g., Bewley et al., ${ }^{5}$ Wei and Freund, ${ }^{8}$ and also documented in Delport et al.: ${ }^{20}$ it relies on the Polak-Ribière conjugate-gradient method ${ }^{21-23}$ combined with the Brent line-search algorithm. ${ }^{21,24}$ The method is a search method with a double iteration loop: at a point $\boldsymbol{\phi}^{(k)}$ in the outer iteration cycle, the conjugate-gradient direction is determined; the next point in the outer iteration $\boldsymbol{\phi}^{(k+1)}$ is then the optimum of the cost functional along this search direction. To find the optimum along the search direction, an iterative line-search algorithm is employed (i.e., the inner iteration cycle). First, an interval which contains a minimum is bracketed using an algorithm based on the mnbrak algorithm; ${ }^{21}$ subsequently, the Brent algorithm ${ }^{21,24}$ is used to iteratively narrow down the interval. For cost-functional evaluations at each point of the inner iteration cycle, separate NavierStokes simulations are required. The determination of the conjugate-gradient direction in the outer cycle requires the gradient $\mathcal{G}^{(k)}$ of the cost functional at the point $\boldsymbol{\phi}^{(k)}$.

To compute the gradient of the cost functional, we need to evaluate the sensitivity of $\mathcal{J}$ at a given point $\boldsymbol{q}(\boldsymbol{x}, T, \boldsymbol{\phi})$ to small changes $\delta \boldsymbol{\phi}$ of the control parameters $\boldsymbol{\phi}$, i.e., the initial perturbations to the mixing layer. The sensitivity is calculated as the Gateau-differential of $\mathcal{J}$ in the point $\boldsymbol{q}(\boldsymbol{x}, T, \boldsymbol{\phi}),{ }^{25}$

$$
\begin{aligned}
\delta \mathcal{J}(\boldsymbol{\phi} ; \delta \boldsymbol{\phi}) & \left.\equiv \frac{\mathrm{d}}{\mathrm{d} \alpha} \mathcal{J}[\boldsymbol{q}(\boldsymbol{x}, T, \boldsymbol{\phi}+\alpha \delta \boldsymbol{\phi})]\right|_{\alpha=0}, \\
& =\int_{\Omega} \frac{\partial J[\boldsymbol{q}(\boldsymbol{x}, T, \boldsymbol{\phi})]}{\partial \boldsymbol{q}} \cdot \delta \boldsymbol{q} \mathrm{d} \boldsymbol{x}
\end{aligned}
$$

with $\delta q(\boldsymbol{x}, T, \boldsymbol{\phi}, \delta \boldsymbol{\phi})$ the sensitivity of the flow solution at the time horizon to a change of $\boldsymbol{\phi}$ in the direction $\delta \boldsymbol{\phi}$. In the current case, $\boldsymbol{\phi}$ contains a large number of degrees of freedom, and the determination $\delta \boldsymbol{q}$ for every possible $\delta \boldsymbol{\phi}$ becomes prohibitive. This problem is circumvented by solving an adjoint problem, ${ }^{5,26-29}$

$$
\begin{aligned}
& \nabla \cdot \boldsymbol{u}^{*}=0, \\
& -\frac{\partial \boldsymbol{u}^{*}}{\partial t}-\boldsymbol{u} \cdot \nabla \boldsymbol{u}^{*}-\boldsymbol{u} \cdot\left(\nabla \boldsymbol{u}^{*}\right)^{T}-\nabla p^{*}-\frac{1}{\operatorname{Re}} \nabla^{2} \boldsymbol{u}^{*}=0 .
\end{aligned}
$$

For a temporal mixing layer, periodic boundary conditions are used in the stream-wise and span-wise directions, and 


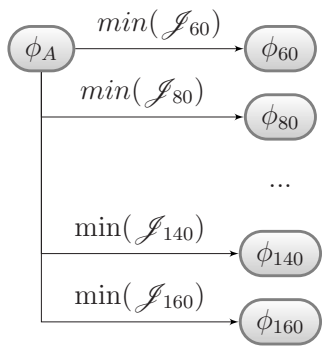

(a)

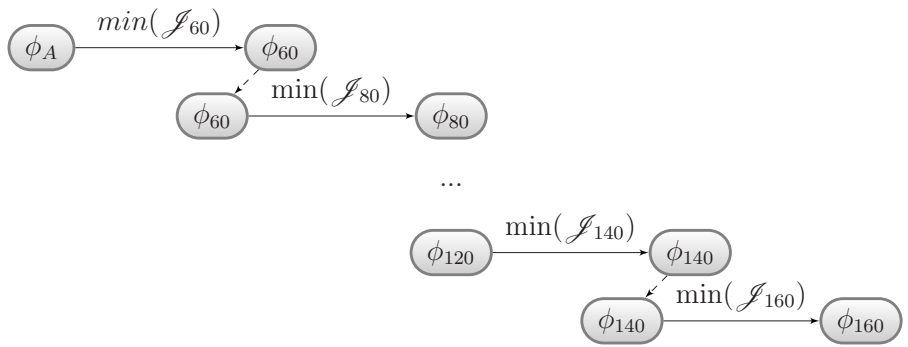

(b)

FIG. 1. Optimization procedure for a set of optimizations toward different time horizons: (a) as used in previous work (Ref. 20) and (b) currently used.

symmetric boundary conditions in the normal direction. ${ }^{20}$ Further, the initial condition for the adjoint equations, which is given at time $T$, is $\boldsymbol{u}^{*}(\boldsymbol{x}, T, \boldsymbol{\phi})=\partial J[\boldsymbol{u}(\boldsymbol{x}, T, \boldsymbol{\phi})] / \partial \boldsymbol{u}$, and the gradient of the cost functional is expressed as ${ }^{20}$

$$
\delta \mathcal{J}[\boldsymbol{q}(\boldsymbol{x}, T, \boldsymbol{\phi}), \delta \boldsymbol{\phi}]=\int_{\Omega} \boldsymbol{u}^{*}(\boldsymbol{x}, 0, \boldsymbol{\phi}) \cdot \delta \boldsymbol{\phi} \mathrm{d} \boldsymbol{x} .
$$

To take the two constraints [Eqs. (6) and (7)] on the control parameters into account, the parameter elimination ${ }^{23}$ and the gradient-projection method ${ }^{22}$ are used, respectively. The parameter elimination method is used for the linear continuity constraint $\nabla \cdot \boldsymbol{\phi}(\boldsymbol{x})=0$. It reduces the possible perturbations $\boldsymbol{\phi}$ on the mixing layer to a subset of linear independent parameters by taking into account the linear constraints imposed by the discretized continuity equations. This subset of linear independent parameters is then optimized using the conjugate-gradient method. The gradient-projection method is used to enforce the energy constraint. The conjugategradient search direction is projected onto the nonlinear energy-constraint surface, such that the constraint is satisfied at all steps during the optimization process. More details can be found in Ref. 20.

For nonconvex optimization, gradient-based procedures may lead to local optima which depend on the starting point of the optimization procedure. In Delport et al. ${ }^{20}$ it was found that local optima emerge with a dependence on the optimization time horizon $T$ that is inconsistent. For instance, when optimization is performed to $\mathcal{J}_{\mathrm{DE}}$, it is expected for two different optimization time horizons $T_{2}>T_{1}$ that $\Delta E\left(T_{2}, \boldsymbol{\phi}_{T_{2}}\right) \geq \Delta E\left(T_{2}, \boldsymbol{\phi}_{T_{1}}\right)$ (cf. Sec. II A). However, in Delport et $a l^{20}{ }^{20}$ we found that this is not always the case while using the conjugate-gradient procedure starting from the same initial guess for the controls (i.e., this was observed for $T_{1}=20, T_{2}=40$, and $\mathcal{J}_{\mathrm{KE}}$ ). Also at the outset of the current study, we were faced with this type of local optima when starting the conjugate-gradient iterations from the same starting point $\boldsymbol{\phi}_{A}$. Therefore, we designed a different optimization procedure, which tries to avoid inconsistent local optima. The procedure is outlined in the two following paragraphs.

In order to get a more consistent characterization of optima as a function of the time horizon $T$, we slightly changed the optimization approach, as schematically shown in Fig. 1. Instead of starting all optimizations for different time horizons directly from $\boldsymbol{\phi}_{A}$, we only start the optimization for the lowest time horizon $(T=60)$ from $\boldsymbol{\phi}_{A}$. Subsequently, the optimal parameter set $\boldsymbol{\phi}_{60}$ is used as a starting point for the optimization to the next time horizon $(T=80)$, and so forth, until the last time horizon $T=160$ (cf. Fig. 1). In this way, $\Delta E\left(T_{2}, \boldsymbol{\phi}_{T_{2}}\right) \geq \Delta E\left(T_{2}, \boldsymbol{\phi}_{T_{1}}\right)$ is guaranteed when $\mathcal{J}_{\mathrm{DE}}$ is minimized (with $T_{1}$ and $T_{2}$ as two subsequent time horizons and $\left.T_{2}>T_{1}\right)$, and $\mathcal{E}\left(T_{2}, \boldsymbol{\phi}_{T_{2}}\right) \geq \mathcal{E}\left(T_{2}, \boldsymbol{\phi}_{T_{1}}\right)$ in case $\mathcal{J}_{\mathrm{DR}}$. This procedure corresponds to a simple continuation approach in the space of optimal solutions. If we presume that $\boldsymbol{\phi}_{T}$ depends continuously on $T$, this is nothing more than a first-order extrapolation of $\boldsymbol{\phi}$, which we use as a starting point for the conjugate-gradient method. In the current work, higher-order continuation approaches were not further investigated.

Notwithstanding the procedure described above, the optimization problem remains nonconvex, and local optima may still exist. In particular, for optimization toward maximum dissipation of energy at the time horizon (case 2), we identified two distinct optima (cf. Sec. IV below for a further discussion on these optima). These optima were found by trying a number of different initial starting points; the two starting points $\boldsymbol{\phi}_{A}(\boldsymbol{x})$ and $\boldsymbol{\phi}_{B}(\boldsymbol{x})$ which led us to these local optima are briefly discussed in Sec. II C.

\section{Case setup and discretization}

We consider a temporal mixing layer in a box with periodic boundary conditions in stream-wise $\left(x_{1}\right)$ and spanwise $\left(x_{2}\right)$ directions, and symmetry conditions in the normal direction $\left(x_{3}\right)$. The initial stream-wise mean velocity corresponds to a tanh-profile (cf. Sec. II A), which imposes a velocity difference between the upper and lower flows of $\Delta U$ $=2$, and an initial vorticity thickness $\delta_{\omega}=2$ [with $\delta_{\omega}$ $\left.\equiv \Delta U /\left.\left(\mathrm{d}\left\langle u_{1}\right\rangle / \mathrm{d} x_{3}\right)\right|_{\max }\right]$. In the current work, all results are normalized using $\delta_{\omega} / 2$ and $\Delta U / 2$ as reference length scale and reference velocity scale. The Reynolds number, based on half of the vorticity thickness, and half of the velocity difference, corresponds to 50, which is similar to the Reynolds number in the study of Vreman et al. ${ }^{13}$ The box size in stream-wise direction corresponds to $L_{1}=123.2=8 \lambda_{1}$, with $\lambda_{1}=15.4$ corresponding with the most unstable wavelength following from linear stability theory. In span-wise direction, the box size $L_{2}=74.04=8 \lambda_{2}$ with $\lambda_{2}=0.6 \lambda_{1}$. Here, $\lambda_{2}$ is the wavelength of the most unstable span-wise perturbation of rollers originating from the Kelvin-Helmholtz instability in the stream-wise direction. ${ }^{16}$ Finally, the box size in the nor- 
mal direction, $L_{3}=240$, is selected sufficiently large to exclude interactions between the mixing region in the center of the domain and the symmetry conditions at the boundaries.

For the discretization of both the standard and adjoint Navier-Stokes equations, a mixed pseudospectral finitevolume code is employed. ${ }^{20,30}$ In the two periodic directions (stream-wise and span-wise), a pseudospectral Fourier discretization is used. Dealiasing of the nonlinear terms is performed using the two thirds dealiasing rule. ${ }^{31}$ The normal direction is discretized using a fourth-order energyconserving staggered finite-volume discretization, ${ }^{32}$ and the locations of normal velocities $u_{3}$ are shifted in the normal direction by half a cell compared to $p, u_{1}$, and $u_{2}$. Continuity is retained by solving a Poisson equation for the pressure using a direct solver.

For the Navier-Stokes simulation, the time integration is performed by a classical four-stage fourth-order accurate Runge-Kutta time integration. Time steps are restricted by setting both the convective and viscous Courant-FriedrichsLewy numbers to 0.2 . For the adjoint simulation, the same time scheme is used but now backward in time. The adjoint flow is solved at the same points in time as in the accompanying nonlinear simulations. This enables the adjoint simulations to use the solution of the preceding Navier-Stokes equations $\boldsymbol{u}\left(\boldsymbol{x}, t_{n}, \boldsymbol{\phi}\right)$ without interpolation. The solution $\boldsymbol{u}\left(\boldsymbol{x}, t_{n}, \boldsymbol{\phi}\right)$ is stored onto disk during the preceding simulation at every grid point and every time step. At the beginning of every Runge-Kutta time step of the adjoint system, the nonlinear solution $\boldsymbol{u}\left(\boldsymbol{x}, t_{n}, \boldsymbol{\phi}\right)$ at step $t_{n}$ is read from the disk, but subsequently kept constant during the Runge-Kutta stages.

All simulations are performed on a $128 \times 128$ $\times 256$ mesh (dealiasing of the nonlinear terms is performed on $192 \times 192 \times 256$ ). In the normal direction, the grid is stretched with a grid-spacing $\Delta x_{3} \sim\left|x_{3}\right|^{1 / 4}$ (this particular stretching is selected based on the observation that, for selfsimilar mixing layers, the Kolmogorov scale $\eta \sim \theta^{1 / 4}$, with $\theta$ the momentum thickness of the layer).

During optimization, we have to ensure that the perturbation field $\boldsymbol{\phi}$ remains sufficiently smooth and well-resolved on the computational grid. In the theory on optimal control of partial differential equations, this is ensured by selecting a sufficiently smooth functional space to which the controls should belong. Elaboration of the gradient, which is the Riesz representation of the derivative (e.g., cf. Ref. 33, p. 98), then leads in practice to the construction of the gradient of the cost functional based on smoothed Gateau differentials (e.g., for a Sobolev space $H^{1}$ this would correspond to an elliptic smoothing operator ${ }^{34}$ ). In the absence of smoothing, it is well documented that optimal controls may become very irregular and "unphysical." 28 In the current work, we approach the issue of constructing a smooth gradient in a more pragmatic way, which does not require the inversion of an elliptic PDE operator. First of all, in the normal direction $\left(x_{3}\right)$, a Gaussian filter was used to filter the gradient, with filter width $10 \Delta x_{3}$, such that $\boldsymbol{\phi}$ remains well-resolved on the computational grid (see Ref. 20 for more details on consistency and convergence of the methodology). In the streamwise and span-wise directions, we elected to restrict the ini- tial perturbations $\boldsymbol{\phi}$ to wavelengths larger or equal to $1 / 16$ th of the box size, which is equivalent to applying a sharp cutoff filter on the gradients. Hence, $\boldsymbol{\phi}$ contains Fourier modes with wave numbers $\left(k_{1}, k_{2}\right)=\left(\alpha 2 \pi / L_{1}, \beta 2 \pi / L_{2}\right)$ with $\alpha, \beta$ $=0, \pm 1, \ldots, \pm 16$ (the mode $\alpha=\beta=0$ is excluded). In this way, the optimal controls remain sufficiently smooth and well-resolved in $x_{1}$ and $x_{2}$ directions. We verified that the eventual controls from our optimization have negligible energy in the vicinity of the Fourier cut-off frequency. Finally, taking all smoothing operations into account, we end up with about $\mathcal{O}\left(10^{5}\right)$ degrees of freedom in the controls, which are to be optimized.

Finally, as previously mentioned in Sec. II B, two starting points for the optimization $\boldsymbol{\phi}^{(0)}$ are employed, $\boldsymbol{\phi}_{A}(\boldsymbol{x})$ and $\boldsymbol{\phi}_{B}(\boldsymbol{x})$. In Fig. 2 the mixing-layer evolution starting from these two initial conditions is displayed. It is appreciated that these initial perturbations (using $E_{0}=10^{-4}$ ) lead to distinctly different evolutions of the temporal mixing layer, both when evaluating $\Delta E$ and $\mathcal{E}$, or when looking at the coherent structures using the $\lambda_{2}$ criterion. The construction of the perturbations $\boldsymbol{\phi}_{A}(\boldsymbol{x})$ and $\boldsymbol{\phi}_{B}(\boldsymbol{x})$ themselves is rather ad hoc, but is briefly added here for the sake of completeness.

The first field $\boldsymbol{\phi}_{A}(\boldsymbol{x})$ is constructed similarly to the initial conditions reported by Moser and Rogers ${ }^{12}$ and Vreman et $a l .{ }^{13}$ The perturbations are a superposition of twodimensional modes [having mode numbers $(\alpha, 0), \alpha$ $=\mp 1 \cdots \mp 16]$ and three-dimensional modes [having mode numbers $(\alpha, \beta), \beta \neq 0]$. All two-dimensional modes use the most unstable eigenfunction in the normal direction for that particular wavelength, following from a linear stability analysis. The phases of the modes are set to zero. For the three-dimensional modes, a Gaussian profile (with $\sigma=3.5$ ) is used in the normal direction for $u_{2}$ and $u_{3}$ (the profile for $u_{1}$ follows from $u_{2}$ and $u_{3}$ using the continuity equation). The total energy is evenly distributed over all modes.

The second set of initial perturbations $\boldsymbol{\phi}_{B}(\boldsymbol{x})$ is composed of broadband random noise for $20 \%$ of its kinetic energy and of low-pass filtered broadband random noise for the remaining $80 \%$ of its total kinetic energy. The noise was generated with the standard pseudorandom number generator of FORTRAN. It imposed the noise field in physical space. Subsequently, the noise was made divergence-free. The lowpass filter employed is a Gaussian filter. Its width is selected such that the transfer function of the filter has a value of 0.5 at the most unstable wave number $(8,0)$ obtained from linear stability theory.

\section{CASE 1: MAXIMIZE DISSIPATED KINETIC ENERGY}

In the current section, the first case is discussed. In Sec. III A, convergence is discussed, results are presented, and prevalent flow features of the optimal solutions are shown. Next, in Sec. III B, optimal solutions are evaluated in terms of their self-similar evolution for long simulation times. In Sec. III C, we further discuss the consequences related to the maximization of total dissipated energy for long time windows. 


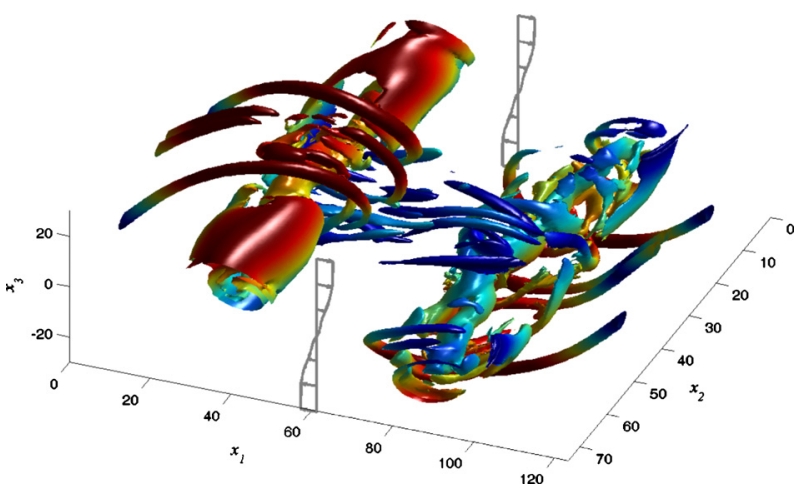

(a) $\phi_{A}$

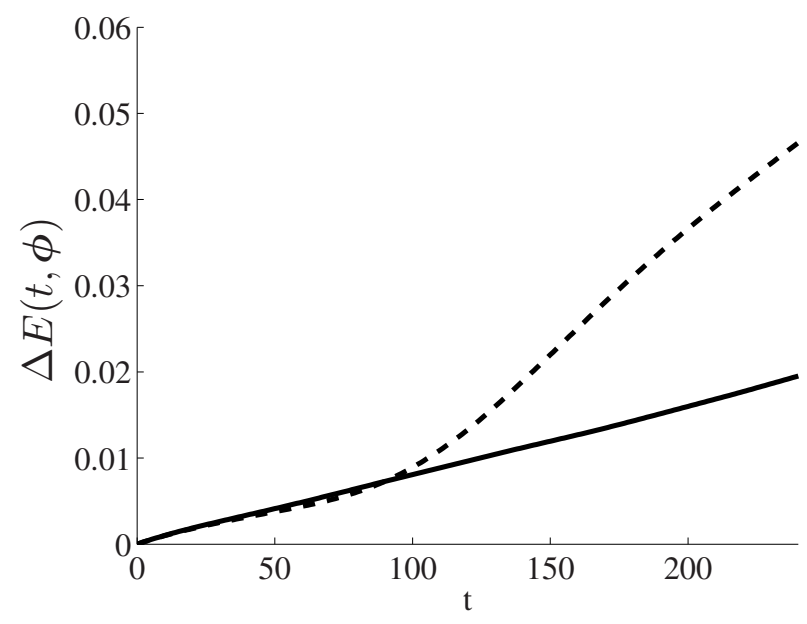

(c) Evolution of $\Delta E$

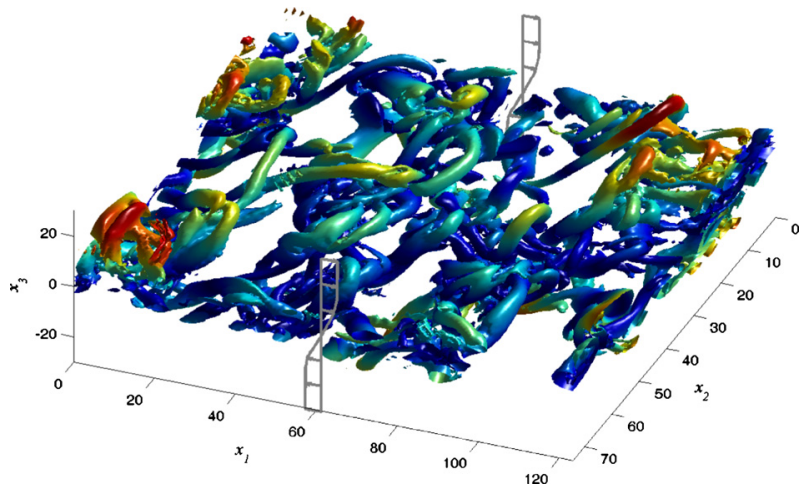

(b) $\phi_{B}$

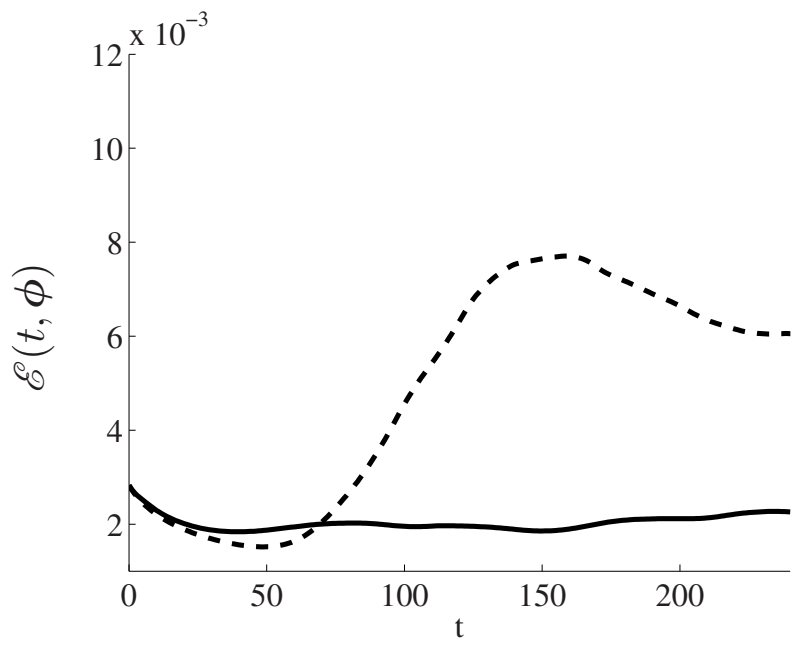

(d) Evolution of $\mathscr{E}$

FIG. 2. (Color online) Mixing-layer evolution starting from two different initial perturbations $\boldsymbol{\phi}_{A}$ and $\boldsymbol{\phi}_{B}$ (with $E_{0}=10^{-4}$ ). [(a) and (b)] $\lambda_{2}$ visualization (Ref. 35) of the velocity field at time $t=120$ (colored online with the distance from the mixing center plane); [-(in gray)]: profiles for $\left\langle u_{1}\right\rangle$. (c) Evolution of the dissipated energy $\Delta E$ and (d) enstrophy $\mathcal{E}$ for initial conditions $(-) \boldsymbol{\phi}_{A}$ and $(--) \boldsymbol{\phi}_{B}$.

\section{A. Optimization results}

Optimization results for the maximization of total dissipated total kinetic energy (i.e., based on $\mathcal{J}_{\mathrm{DE}}$ ), and using $\boldsymbol{\phi}_{A}$ as an initial guess, are now presented in Fig. 3 for both energy levels $E_{0}=10^{-5}$ and $10^{-4}$. In Figs. 3(a) and 3(c), the convergence history as a function of the number of conjugate-gradient (outer) iterations is presented. We use a convergence criterion based on a relative improvement of the cost functional of $10^{-10}$ normalized with the total kinetic energy at the time horizon. As shown in Figs. 3(a) and 3(c), not all cases are converged up to this criterion; instead, some were stopped after 200 iterations in order to limit the computational costs (i.e., we recall that one conjugate-gradient iteration requires one adjoint Navier-Stokes simulation for the gradient calculation and about eight Navier-Stokes simulations for the line-search algorithm).

The evolution of the total dissipated energy $\Delta E\left(t, \boldsymbol{\phi}_{T}\right)$ is displayed for different time horizons in Figs. 3(b) and 3(d), respectively, for $E_{0}=10^{-5}$ and $10^{-4}$. A large increase of the total dissipated energy for the optimized cases is appreciated when compared to the evolution with $\boldsymbol{\phi}_{A}$ (also displayed in the figures). Optimizations starting from different initial guesses (e.g., $\boldsymbol{\phi}_{B}$ ) do not lead to better optima for $\mathcal{J}_{\mathrm{DE}}$ and are not further discussed here.

When looking at Figs. 3(b) and 3(d), the first important observation is that, for $T_{2}>T_{1}$, the corresponding optimal energy levels $\Delta E\left(T_{1}, \boldsymbol{\phi}_{T_{2}}\right) \approx \Delta E\left(T_{1}, \boldsymbol{\phi}_{T_{1}}\right)$. Hence, for the time horizons investigated here, the optimal solution for a time horizon $T$ is also optimal for all time horizons smaller than $T$, in particular, we find that $\boldsymbol{\phi}_{160}$ is optimal for 60 $\leq T \leq 160$.

To further analyze this, it is useful to focus on the maximum dissipated total kinetic energy for each time horizon $\Delta E\left(T, \boldsymbol{\phi}_{T}\right)$ as a function of the time horizon $T$. This is not anymore the evaluation of the time evolution of one optimum [as, e.g., expressed by $\left.\Delta E\left(t, \boldsymbol{\phi}_{T}\right)\right]$, but rather the evolution of the optima as a function of the optimization time horizon, evaluated at time $t=T$. Figure 4 shows that $\Delta E\left(T, \boldsymbol{\phi}_{T}\right)$ is a monotonic function that increases with $T$. 


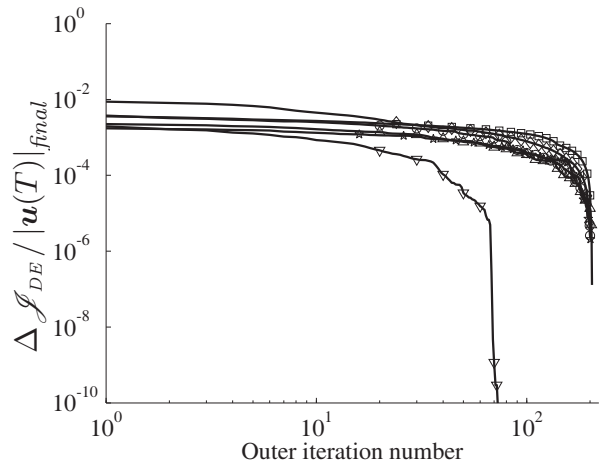

(a) $E_{0}=10^{-5}$, starting point $\phi_{A}$

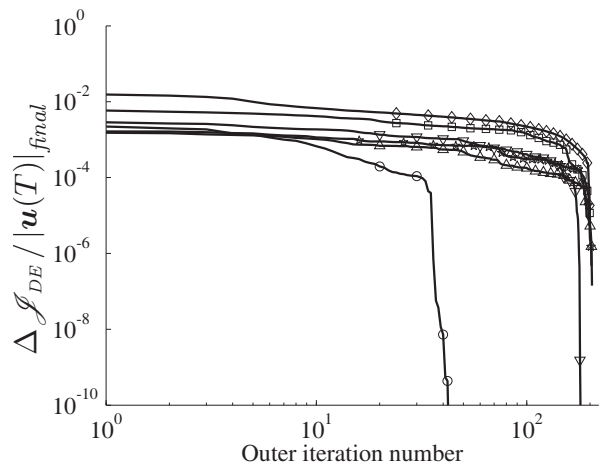

(c) $E_{0}=10^{-4}$, starting point $\phi_{A}$

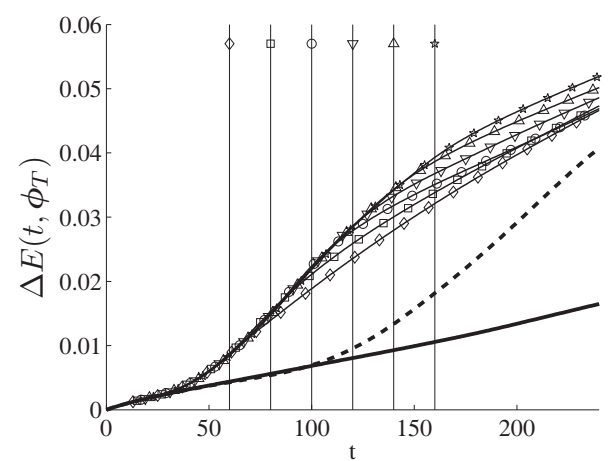

(b) $E_{0}=10^{-5}$, starting point $\phi_{A}$

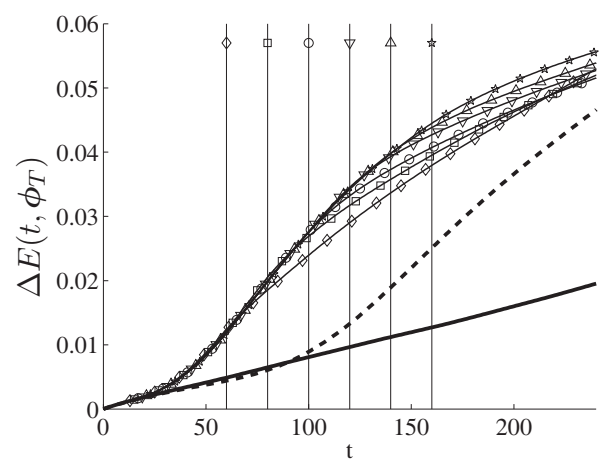

(d) $E_{0}=10^{-4}$, starting point $\phi_{A}$

FIG. 3. Optimization results for $\mathcal{J}_{\mathrm{DE}}$ starting from $\boldsymbol{\phi}_{A}$. [(a) and (c)] Convergence history for the different time horizons $\left[\right.$ with $\left.\Delta \mathcal{J}_{\mathrm{DE}}=\mathcal{J}_{\mathrm{DE}}(i t)-\mathcal{J}_{\text {final }}\right]$ normalized with the kinetic energy at the time horizon when the final parameters are used $|\boldsymbol{u}(T)|_{\text {final }}$. $[(\mathrm{b})$ and (d)] Evolution of the total dissipated energy $\Delta E\left(t, \boldsymbol{\phi}_{T}\right)$ as a function of time for different time horizons. Symbols in (a)-(d): $T=60(\diamond), T=80(\square), T=100(\bigcirc), T=120(\nabla), T=140(\triangle), T=160(\star)$. Additional lines in (b) and (d): evolution of the total dissipated energy for initial condition $\boldsymbol{\phi}_{A}(-)$ and $\boldsymbol{\phi}_{B}(--)$; vertical lines are drawn at the different optimization time horizons.

Further, $\Delta E\left(T, \boldsymbol{\phi}_{T}\right)=\Delta E\left(T, \boldsymbol{\phi}_{160}\right)$, with $T=160$ the longest optimization time horizon included in the current study. $\Delta E\left(t, \boldsymbol{\phi}_{T}\right)$ may also be expressed as

$$
\Delta E\left(t, \boldsymbol{\phi}_{T}\right)=2 \nu \int_{0}^{t} \mathcal{E}\left(t^{\prime}, \boldsymbol{\phi}_{T}\right) \mathrm{d} t^{\prime},
$$

with $\mathcal{E}\left(t, \boldsymbol{\phi}_{T}\right)$ the volume-averaged enstrophy evolution starting from the $\mathcal{J}_{\mathrm{DE}^{-}}$-optimal perturbations $\boldsymbol{\phi}_{T}$. Therefore, $\Delta E\left(T_{1}, \boldsymbol{\phi}_{T_{2}}\right) \approx \Delta E\left(T_{1}, \boldsymbol{\phi}_{T_{1}}\right)$ for $T_{2}>T_{1}$ requires that the integrals of $\mathcal{E}\left(t, \boldsymbol{\phi}_{T_{1}}\right)$ and $\mathcal{E}\left(t, \boldsymbol{\phi}_{T_{2}}\right)$ are equal when integrated

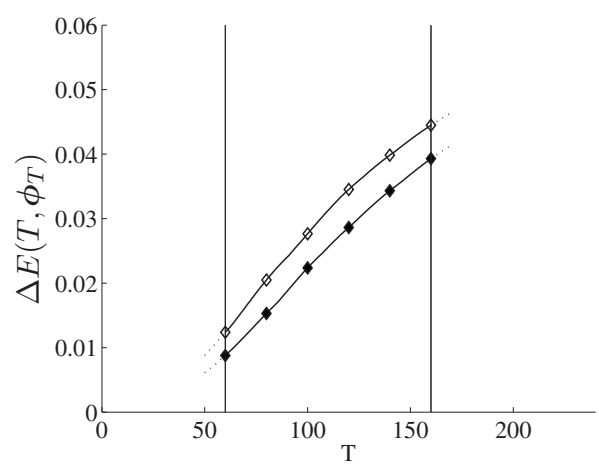

FIG. 4. Maximal total dissipated energy as a function of time horizon $T$ for flows optimized toward $\mathcal{J}_{\mathrm{DE}}$. $(-\diamond) E=10^{-5}$ and $(-\diamond) E=10^{-4}$. from 0 to $T_{1}$. This is illustrated in Fig. 5(a), where the enstrophy evolution of flows optimized to $\mathcal{J}_{\mathrm{DE}}$ (and $E_{0}=10^{-4}$ ) is shown. In this figure, it is now observed for all optima (except for $T=60$ and 80 ) that the enstrophy evolutions collapse with a peak around $t \approx 70$. Moreover, the longer the optimization time horizons, the longer optimal solutions manage to sustain a high enstrophy level after the peak (i.e., for $T=80$, the enstrophy drastically drops down starting at $t \approx 70$, for $T=100$ this occurs at $t \approx 90$, for $T=120$ at $t=110$, etc.).

Further, we also display for the different optimization cases the evolution of the turbulent kinetic energy in Fig. 5(b). It is appreciated that the turbulent kinetic energy increases significantly compared to its initial level, i.e., 0.5 $\times 10^{-4}$ initially compared to, e.g., 0.015 at $t=60$. The latter energy level corresponds to turbulent fluctuations with an amplitude of approximately $10 \%$ of the reference velocity $(\Delta U / 2)$. Taking into account that all optimization time horizons at which cost-functional evaluations are defined are larger (or equal) than $t=60$, this clearly illustrates that the current optimization is in the nonlinear regime, with amplitudes which are too large to rely on linear methods. ${ }^{18}$

When comparing $\Delta E\left(T, \boldsymbol{\phi}_{T}\right)$ for $E_{0}=10^{-5}$ and $E_{0}=10^{-4}$ in Fig. 4, we observe a straightforward effect of the control energy: more energy in the initial perturbations yields higher 


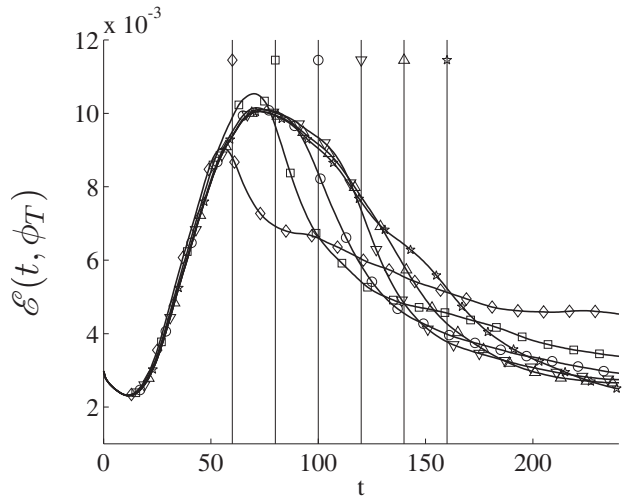

(a)

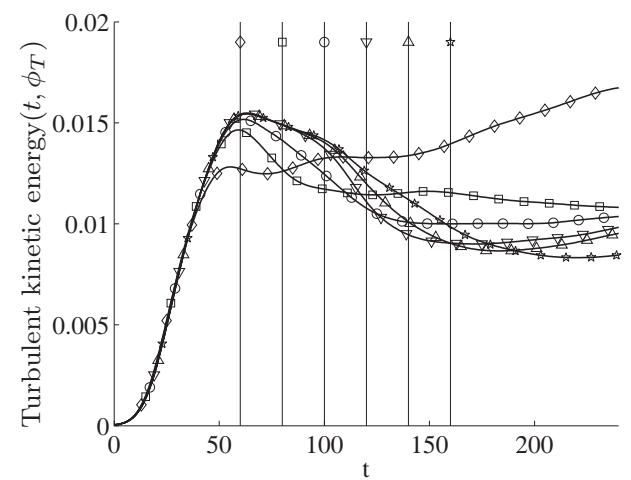

(b)

FIG. 5. Evolution of the enstrophy (a) and the turbulent kinetic energy (b) in time for flows optimized toward $\mathcal{J}_{\text {DE }} . T=60(\diamond), T=80(\square), T=100(\bigcirc), T$ $=120(\nabla), T=140(\triangle), T=160(\star)$ for $E_{0}=10^{-4}$.

dissipated energy for all optimization time horizons. Apart from this, we did not observe any substantial differences with respect to the observations discussed above. When looking at the shape of turbulent structures which emerge in the evolution of the mixing layer, small differences are observed, as discussed next.
The flow structures of the shear layer optimized to $\mathcal{J}_{\mathrm{DE}}$ with time horizon $T=160$ are now visualized in Fig. 6 using the $\lambda_{2}$ criterion. $^{35}$ The structures observed for other optimization time horizons are very similar and are not further shown here. In Fig. 6 (right), the coherent structures at the time horizon $t=160$ are displayed. It is appreciated that the
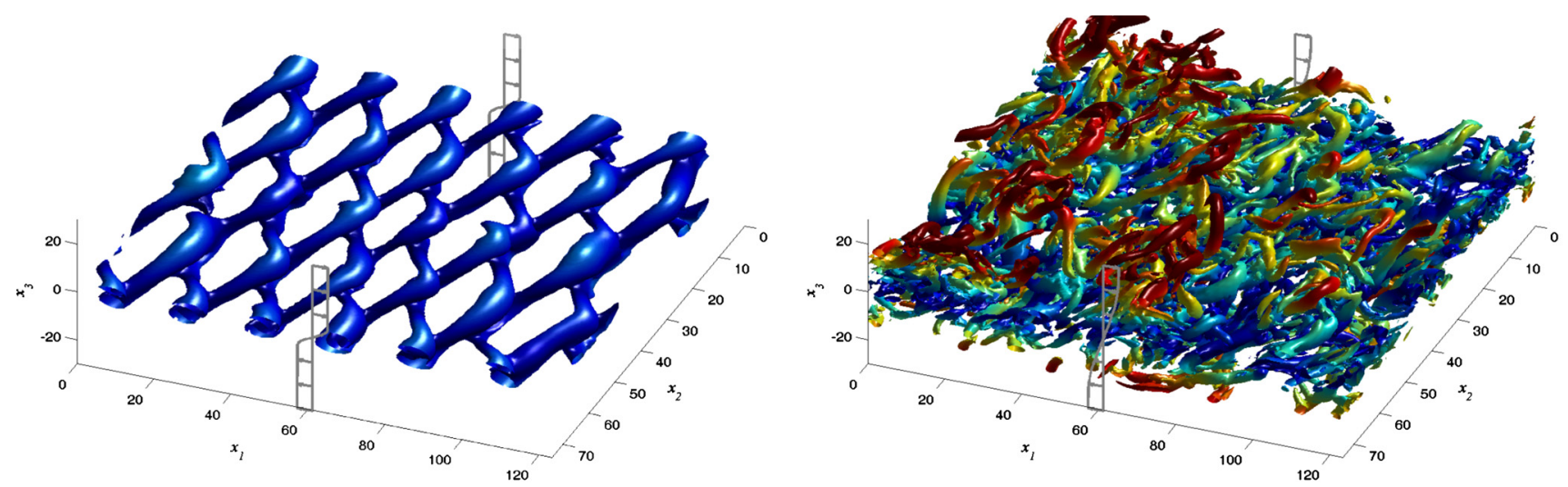

(a) $E_{0}=10^{-5}, T=160$
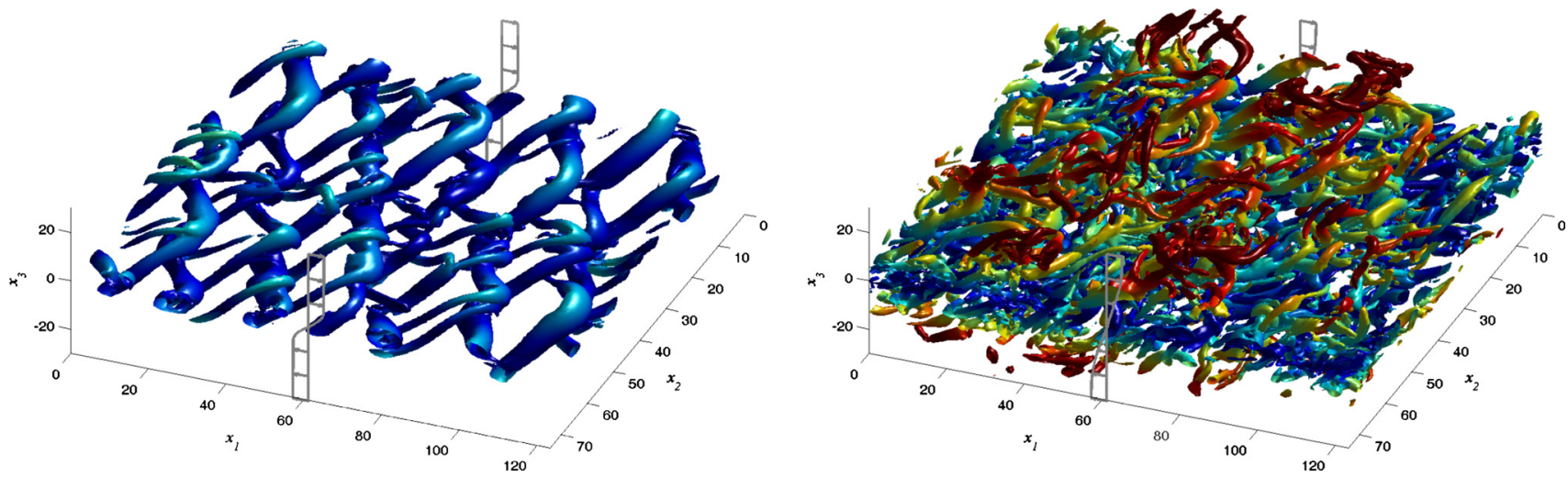

(b) $E_{0}=10^{-4}, T=160$

FIG. 6. (Color online) $\lambda_{2}$ visualization of the velocity field for parameters optimized toward $\mathcal{J}_{\text {DE }}$ (colored online with the distance from the mixing center plane); profile $\left\langle u_{1}\right\rangle$ (一); visualization of $t=30$ (left) and $t=160$ (right). 


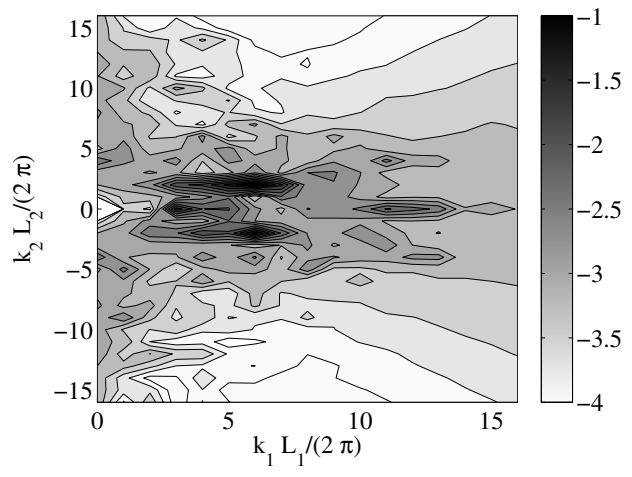

(a) $E_{0}=10^{-5}, T=160$

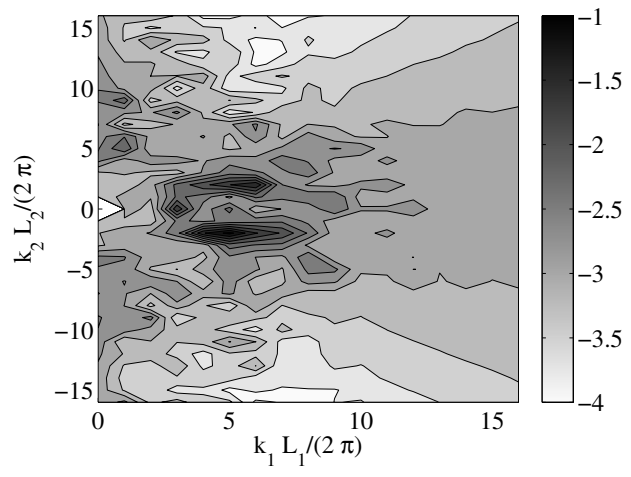

(b) $E_{0}=10^{-4}, T=160$

FIG. 7. Energy distribution over the modes of the optimal initial perturbations (at $t=0$ ) optimized to time horizon $T=160$ and colored by $\log \left[E_{\text {mode }} /\left(E_{0} \Delta U^{2} / 8\right)\right]$. Energy is integrated over $x_{3}$.

shear layer is fully turbulent, dominated by small-scale turbulent structures. In Fig. $6, \lambda_{2}$ results are also shown at $t$ $=30$ to evaluate the coherent structures in the early transition phase of the shear layer. The evaluation of $\lambda_{2}$ at $t=0$ does not yield any meaningful results.

It is appreciated in Fig. 6 (left) that the coherent structures at $t=30$ for $E_{0}=10^{-5}$ form a diamond-shaped vortex pattern. Figure 7, displaying the energy distribution of the optimal perturbations (at $t=0$ and with optimization time horizon $T=160)$, illustrates that these diamonds originate from a high initial energy concentration in the $(6, \pm 2)$ mode. This mode has a wavelength $\left(8 \lambda_{1} / 6,8 \lambda_{2} / 2\right)$ (with $\lambda_{1}=15.4$ and $\lambda_{2}=0.6 \lambda_{1}$, cf. Sec. II C). The coherent structures at $t=30$ for $E_{0}=10^{-4}$ look similar, but with some additional features imposed on top of the smooth diamond shapes observed for $E_{0}=10^{-5}$. Also in Fig. 7, it is observed for $E_{0}=10^{-4}$ that the energy is mainly concentrated at the same modes.

Similar diamond-shaped vortex structures have also been observed in experiment of a forced mixing layer by Nygaard and Glezer (Fig. 5 in Ref. 36). They tested the impact of a span-wise array of surface heaters, placed on a splitter plate, on the subsequent flow evolution. With an adequate phase difference between the heaters, diamond-shaped structures are obtained. Collis et al. ${ }^{37}$ showed that these structures may also be observed in an incompressible temporal mixing layer when a pair of oblique disturbances is used for the initial condition, which is similar to our observation. They also showed that this flow behavior increases mixing compared to standard rib/roller flows. ${ }^{37}$

It is also interesting to compare the current diamondshaped structures to the vortex patterns emerging in classical transition to turbulence reported for mixing layers. In the latter case, random perturbations trigger the KelvinHelmholtz instability (with wavelength $\lambda_{1}=15.4$ in the current study), which grows exponentially at first, and subsequently saturates into two-dimensional Kelvin-Helmholtz rollers. These two-dimensional vortex structures may pair into larger rollers, but in the presence of three-dimensional disturbances they are also subject to secondary threedimensional instabilities, which lead to the formation of stream-wise vorticity and transition to turbulence. The two main secondary instabilities are a core-centered elliptic insta- bility, with most unstable wavelength in the $x_{2}$ direction around $^{16,17} 0.5 \lambda_{1}-0.7 \lambda_{1}$, and a braid-centered hyperbolic instability, with most unstable wavelength (in $x_{2}$ ) around ${ }^{17}$ $0.2 \lambda_{1}$. Our current, optimized perturbations [dominated by $(6, \pm 2)$ modes] clearly follow a different transition path, and the dominant wavelengths are much larger, i.e., by $30 \%$ in the stream-wise direction (compared to the KelvinHelmholtz wavelength) and by a factor of 4-12 in the spanwise direction (compared to wavelengths of the corecentered and braid-centered instabilities, respectively).

Based on the observations in Figs. 6 and 7, we investigate how many modes are essential for the optimal evolution (leading to maximal dissipated energy) of the mixing layer. To this end, for $E_{0}=10^{-5}$ initial conditions are constructed by only taking the first $n$ dominant modes from Fig. 7 (based on the energy per mode), and we monitor the total energy dissipation for different values of $n$. For $n=2$ [only modes $(6,2)$ and $(6,-2)]$, it is found that the total dissipation reaches $66 \%$ of the optimal dissipation. For $n=4$, the total energy dissipated corresponds to $74 \%$ of the optimal value. For $n=8$, we find a total dissipation that is only slightly higher (75\%). Hence, using only four modes, up to $75 \%$ of the energy dissipation realized by optimal control may be recovered.

\section{B. Self-similarity}

Here, the evolution of the optimized mixing layers for longer simulation times is monitored beyond the time horizon for which the initial controls are optimized. For all cases, self-similarity is observed.

For temporal mixing layers, self-similarity is characterized by linear growth of the momentum thickness $(\theta$ $\left.=\int\left[1 / 4-\left(\left\langle u_{1}\right\rangle / \Delta U\right)^{2}\right] \mathrm{d} x_{3}\right)$ in time. Also, turbulent kinetic energy grows linearly in time, and the ratio between the total production and the total dissipation of turbulent kinetic energy is constant. Small turbulent scales in the flow are in equilibrium, which can, e.g., be verified by looking at the balance between enstrophy production and dissipation. Various other diagnostics can be investigated, such as the collapse of mean velocity profiles, and Reynolds stresses when appropriately scaled with $\theta$ and $\Delta U$, etc. More details are, e.g., found in Refs. 3, 4, 38, and 39. 


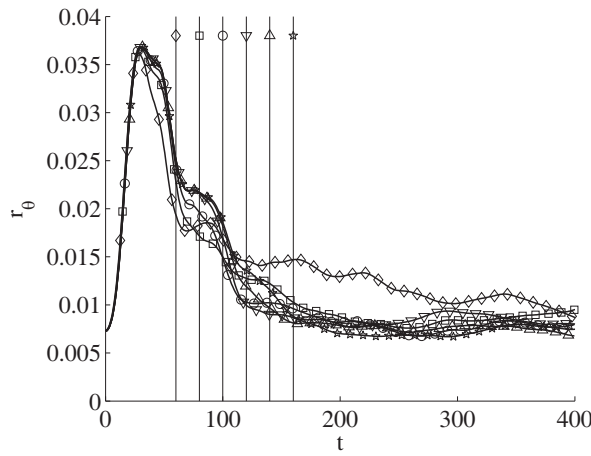

FIG. 8. Evolution of the momentum thickness growth rate in time for flows optimized toward $\mathcal{J}_{\mathrm{DE}}, E_{0}=10^{-4} . T=60(\diamond), T=80(\square), T=100(\bigcirc), T$ $=120(\nabla), T=140(\triangle), T=160(\star)$.

In Fig. 8, the growth rate $r_{\theta} \equiv \mathrm{d} \theta / \mathrm{d} t / \Delta U$ of the mixinglayer momentum thickness is displayed for $E_{0}=10^{-4}$ and flow optimized to $\mathcal{J}_{\mathrm{DE}}$. It is observed that optimized results roughly reach a constant growth rate for $t \geq 160$ with values between 0.007 and 0.01 . This range is a bit lower than the value of 0.014 obtained in the study of Rogers and Moser. ${ }^{3}$

In Fig. 9(a), the ratio between production and dissipation of turbulent kinetic energy at the mixing-layer center is shown. They are, respectively, defined as (see, e.g., Ref. 39)

$$
\mathcal{P}=-\left.\frac{1}{L_{1} L_{2}} \int\left(u_{1}^{\prime} u_{3}^{\prime} \frac{\partial\left\langle u_{1}\right\rangle}{\partial x_{3}}\right)\right|_{x_{3}=0} \mathrm{~d} x_{1} \mathrm{~d} x_{2}
$$

and

$$
\mathcal{D}=\frac{1}{L_{1} L_{2}} \int 2 \nu(s: s)_{x_{3}=0} \mathrm{~d} x_{1} \mathrm{~d} x_{2},
$$

with $s=\left[\nabla \boldsymbol{u}^{\prime}+\left(\nabla \boldsymbol{u}^{\prime}\right)^{T}\right] / 2$ the shear-stress tensor related to the fluctuating velocity $\boldsymbol{u}^{\prime}=\boldsymbol{u}-\langle\boldsymbol{u}\rangle$ (and $\langle\boldsymbol{u}\rangle$ the velocity averaged over $x_{1}-x_{2}$ planes). For all cases, we observe a ratio $\mathcal{P} / \mathcal{D} \approx 1.35$, evaluated in the mixing layer's self-similar region. This corresponds well to the ratio of 1.4 , which was observed by Rogers and Moser. ${ }^{3}$

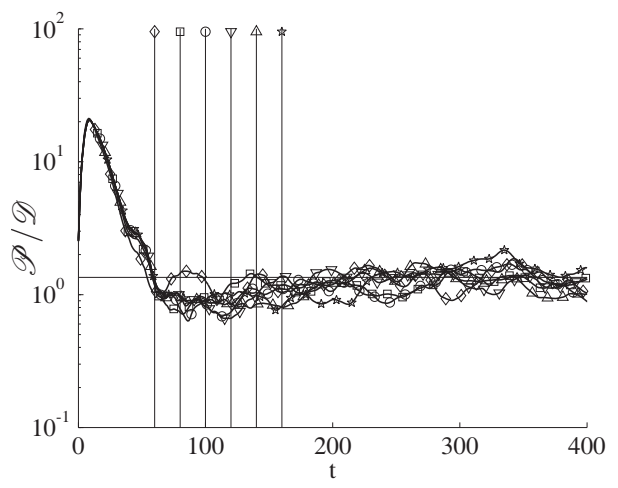

(a) $\mathscr{P} / \mathscr{D}$
To investigate the equilibrium of the small-scale turbulence, the ratio of enstrophy production to enstrophy dissipation is displayed in Fig. 9(b). Production and dissipation of enstrophy are defined by (cf. Ref. 39)

$$
\mathcal{P}_{\mathcal{E}}=\frac{1}{\Omega} \int_{\Omega}(\omega \cdot \nabla \boldsymbol{u}) \cdot \boldsymbol{\omega} \mathrm{d} \boldsymbol{x}
$$

and

$$
\mathcal{D}_{\mathcal{E}}=\frac{1}{\Omega} \int_{\Omega} \nu(\nabla \boldsymbol{\omega}: \nabla \boldsymbol{\omega}) \mathrm{d} \boldsymbol{x} .
$$

It is appreciated that a ratio of one is reached in all cases, and we observe (not shown here) a constant enstrophy level of $3.7 \times 10^{-3}$ for all cases; this value is closely corresponding to the values reported in Balaras et al. ${ }^{4}$ and Rogers and Moser. ${ }^{3}$ The Taylor-Reynolds number in our optimized cases, measured in the mixing center plane, ranges between $\operatorname{Re}_{\lambda} \approx 40$ (at $t=160$ ) and $\mathrm{Re}_{\lambda} \approx 55$ (at $t=400$ ).

The time at which the self-similar region starts is difficult to identify precisely. We look at the emergence of a constant growth rate, at the collapse of normal Reynoldsstress profiles (not shown here), and at a constant $\mathcal{P} / \mathcal{D}$ ratio, and find that self-similarity starts at $t \approx 160-200$ for all cases.

\section{Discussion}

Results in Figs. 8 and 9 show self-similarity for all optimized cases when the simulations are sufficiently continued beyond the optimization time horizon, i.e., at $t=200$. These observations are further discussed here.

The Reynolds number based on momentum thickness $\operatorname{Re}_{\theta} \equiv \theta \Delta U / \nu$ at which a self-similar state emerges is significantly lower than the values reported in earlier studies. We find in our optimized cases $\operatorname{Re}_{\theta} \approx 350$ at the onset of selfsimilarity (evaluated at $t=200$ ), which should be compared to $\mathrm{Re}_{\theta} \approx 1900$ found by Balaras et al. ${ }^{4}$ and $\mathrm{Re}_{\theta} \approx 1440$ found by Rogers and Moser. ${ }^{3}$ Hence, in the current study, Reynolds numbers at the start of the self-similar region are a factor of

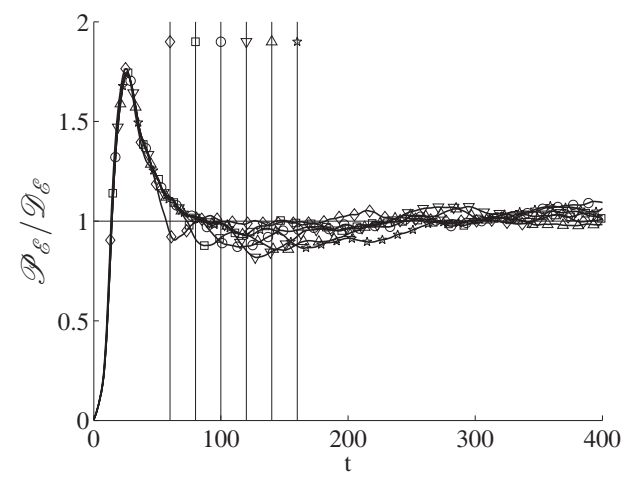

(b) $\mathscr{P}_{\mathscr{E}} / \mathscr{D}_{\mathscr{E}}$

FIG. 9. (a) Evolution of $\mathcal{P} / \mathcal{D}$ in the center of the layer in time. Horizontal line at 1.35. (b) Evolution of the rate of enstrophy production to enstrophy dissipation in time. Horizontal line at 1.0. [(a) and (b)] Flows optimized toward $\mathcal{J}_{\text {DE }}$ with $T=60(\diamond), T=80(\square), T=100(\bigcirc), T=120(\nabla), T=140(\triangle), T$ $=160(\star), E_{0}=10^{-4}$. 
4-5 lower. The Taylor-Reynolds number at the onset of selfsimilarity corresponds to $\operatorname{Re}_{\lambda} \approx 40$. Consequently, compared to uncontrolled initial conditions, optimization to $\mathcal{J}_{\mathrm{DE}}$ leads to a significant speedup of the transition of the mixing layer to a self-similar state, which is reached at much lower Reynolds numbers.

In Sec. III A, it was observed that the optimal controls $\boldsymbol{\phi}_{160}$ are also optimal for $60 \leq T \leq 160$. Moreover, the shearlayer evolution of this optimum displays a fast transition to a self-similar regime at Reynolds numbers which are significantly lower than previously reported. This suggests that optimal control maximizing the total dissipation leads to a fast transition to self-similarity.

When looking at the evolution of dissipation in time of the optimal solutions, we find that they all peak around $t$ $=70$ (cf. Fig. 5). Afterward, the level of dissipation decreases and eventually falls back in the self-similar region (starting at $t \approx 160-200$ ) to a constant level which is the same for all cases, and which also corresponds to enstrophy levels of other uncontrolled self-similar mixing layers. ${ }^{3,4}$ Based on these elements, we surmise that control actions which optimize total dissipated energy do not influence the self-similar region. This also implies that for $T \rightarrow \infty$ this type of controls may become ineffective, i.e., the transition part which can be controlled becomes negligible in the total integral.

Obviously, the optima $\boldsymbol{\phi}_{T}$ discussed here may correspond to a branch of local optima, i.e., other optima (which we did not find) might exist which do not comply with the above observations. Moreover, limitations on computational resources did not allow optimizations with $T>160$, such that time horizons extending significantly into the self-similar region are currently not feasible. Therefore, in a next step to investigate control of dissipation in a shear layer, we consider a second optimization case, which maximizes the dissipation rate at the time horizon (or, equivalently, the enstrophy). For longer time horizons (up to $T=160$ ), the "optimal" level of enstrophy compared to the self-similar level is elaborated, and effects of the controls on the transition to selfsimilarity are quantified. This is elaborated in Sec. IV.

\section{CASE 2: MAXIMIZE DISSIPATION RATE AT TIME HORIZON}

Maximization of the kinetic-energy dissipation rate at the time horizon is now considered. In Sec. IV A, optimization results are presented. Subsequently, in Sec. IV B, a discussion is presented, including the effects of optimal controls on the transition to self-similarity.

\section{A. Optimization results}

As in Sec. III, optimization is performed for energy levels $E_{0}=10^{-4}$ and $E_{0}=10^{-5}$ starting from point $\boldsymbol{\phi}_{A}$. Next to that, for one case $\left(\mathcal{J}_{\mathrm{DR}}\right.$ and $\left.E_{0}=10^{-4}\right)$, the iterative procedure is started from a different starting point $\boldsymbol{\phi}_{B}$, again using six different time horizons, leading to a different branch of optima, further discussed below.

Optimization results for $\mathcal{J}_{\mathrm{DR}}$ and $E_{0}=10^{-4}$ starting from $\boldsymbol{\phi}_{A}$ are presented in Fig. 10. In Fig. 10(a), the convergence history as a function of the number of conjugate-gradient (outer) iterations is presented. We used a convergence criterion based on a relative improvement of the cost functional of $10^{-10}$. It is appreciated that optimizations to all time horizons converge well with respect to this criterion, except for the optimization to $T=120$, which was stopped after 200 iterations for resource reasons.

First of all, in Fig. 10(b) the enstrophy evolution $\mathcal{E}\left(t, \boldsymbol{\phi}_{T}\right)$ is displayed for the different time horizons. A large increase of the enstrophy for the optimized cases is appreciated when compared to the enstrophy evolution of $\boldsymbol{\phi}_{A}$ (also displayed in the figure). For low time horizons ( $T=60$ and 80$)$, the enstrophy evolution strongly peaks near the time horizon for which it is optimized, followed by a sharp decline of enstrophy for $t>T$. For longer time horizons $(T \geq 100)$, this is not the case any more. Now, peaks around $t=80$ are observed followed with a gradual decline up to the time horizon $(t=T)$. For $t$ $>T$, again a sharper decline in enstrophy is seen. In Fig. $11(\mathrm{c})$, results for the lower energy level $E_{0}=10^{-5}$ are also presented (convergence for all time horizons up to the relative convergence criterion of $10^{-10}$ is reached-not shown here). It is appreciated that also for a lower energy level of the controls, a significant improvement of the enstrophy at the optimization time horizon can be obtained compared to the unoptimized starting point $\boldsymbol{\phi}_{A}$. Next to that, the same trends may be observed as for $E_{0}=10^{-4}$.

The evolution of the enstrophy starting from the initial field $\boldsymbol{\phi}_{B}$ (without any optimization) is also displayed in Fig. 10(b) [and Fig. 10(c)]. We observe that this (unoptimized) solution yields a higher level of enstrophy than the optimal solutions $\mathcal{E}(t, T)$ for $T>120$ optimized using the initial guess $\boldsymbol{\phi}_{A}$. Consequently, for $T>120$, the optima obtained using $\boldsymbol{\phi}_{A}$ as an initial starting point are local optima.

To further investigate this, a set of optimizations runs is started from initial point $\boldsymbol{\phi}_{B}{ }^{40}$ For the sake of simple nomenclature, we refer to the set of optima coming from the initial starting point $\boldsymbol{\phi}_{A}$ as "group A," and similarly from $\boldsymbol{\phi}_{B}$ as "group B." In Fig. 11(a), the evolution of the enstrophy is shown for the optima from group B with $E_{0}=10^{-4}$. As is now appreciated, the optimization results based on $\boldsymbol{\phi}_{B}$ provide a large increase of enstrophy compared to the unoptimized $\boldsymbol{\phi}_{B}$ simulation and are considerably better than optima from group A for $T>120$.

To further collect all information on the optimal control of the dissipation rate into one plot, for all cases we display in Fig. 12 the maximum enstrophy for each time horizon $\mathcal{E}\left(T, \boldsymbol{\phi}_{T}\right)$ as a function of the time horizon $T$ [instead of $\left.\mathcal{E}\left(t, \boldsymbol{\phi}_{T}\right)\right]$. Looking first at the maximum enstrophy using an energy level $E_{0}=10^{-4}$ for the controls, a difference between optimizations in group A and group B is noted. For $T<100$, optimizations in group A yield the highest enstrophy $\mathcal{E}\left(T, \boldsymbol{\phi}_{T}\right)$, while the inverse holds for $T>100$. Overall, combining the best optima of group A and group B, we find that optimal controls lead to a maximal enstrophy larger than $8.6 \times 10^{-3}$, which is almost three times higher than the eventual equilibrium level in the self-similar region (cf. Sec. IV B for further discussion). Using the best optima from groups A and $\mathrm{B}$, a slight drop in the level of maximal enstrophy (about $10 \%)$ is observed for longer time horizons compared to shorter optimization time horizons. Figure 12 also shows 


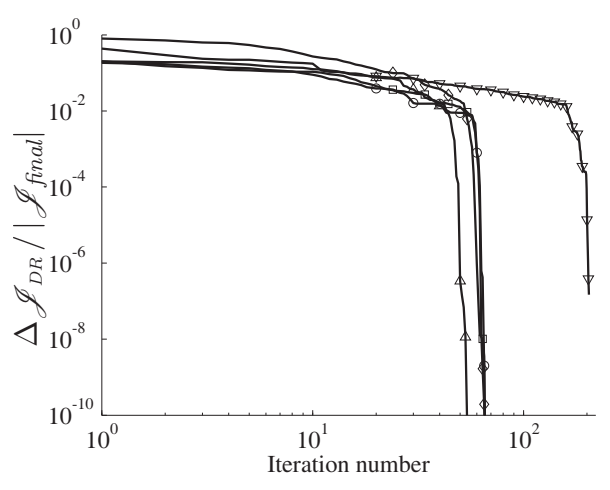

(a)

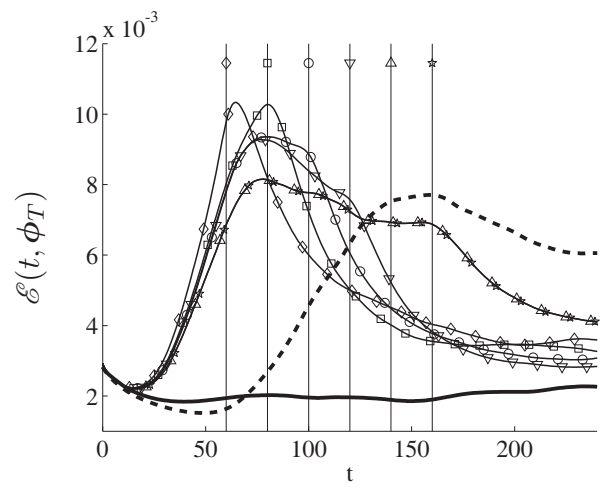

(b)

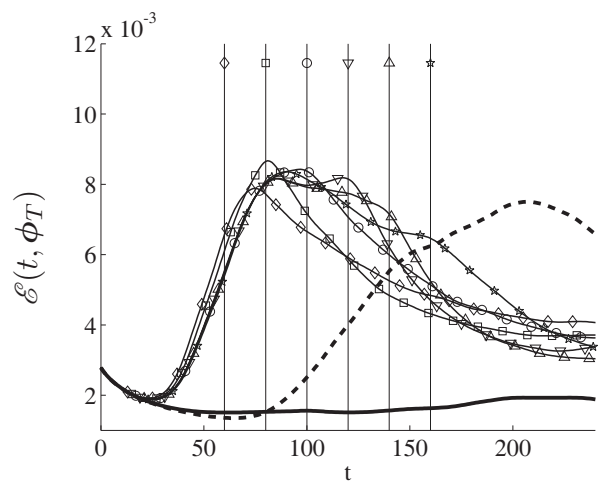

(c)

FIG. 10. Optimization results for $\mathcal{J}_{\mathrm{DR}}$ starting from $\boldsymbol{\phi}_{\mathrm{A}}$. (a) Convergence history for the different time horizons and $E_{0}=10^{-4}$ [with $\left.\Delta \mathcal{J}_{\mathrm{DR}}=\mathcal{J}_{\mathrm{DR}}(i t)-\mathcal{J}_{\text {final }}\right]$. (b) Evolution of the enstrophy $\mathcal{E}\left(t, \boldsymbol{\phi}_{T}\right)$ as a function of time for different time horizons and $E_{0}=10^{-4}$. (c) Evolution of $\mathcal{E}\left(t, \boldsymbol{\phi}_{T}\right)$ for $E_{0}=10^{-5}$. Symbols in (a)-(c): $T=60(\diamond), T=80(\square), T=100(\bigcirc), T=120(\nabla), T=140(\triangle), T=160(\star)$. Additional lines in (b) and (c): enstrophy evolution of initial condition $\boldsymbol{\phi}_{A}(-)$ and $\boldsymbol{\phi}_{B}(--)$; vertical lines are drawn at the different optimization time horizons.

$\mathcal{E}\left(T, \boldsymbol{\phi}_{T}\right)$ for $E_{0}=10^{-5}$. Comparing this to the maximum enstrophy of control-energy level $E_{0}=10^{-4}$ (both from group A), we observe for short time horizons $(T<100)$ that the higher energy level realizes a higher level of enstrophy. For longer time horizons, this difference disappears, and enstrophy levels for both cases are roughly equal.

Finally, the coherent structures associated with the different solutions are elaborated in Fig. 13 using the $\lambda_{2}$-criterion. ${ }^{35}$ We focus on the structures during early tran-

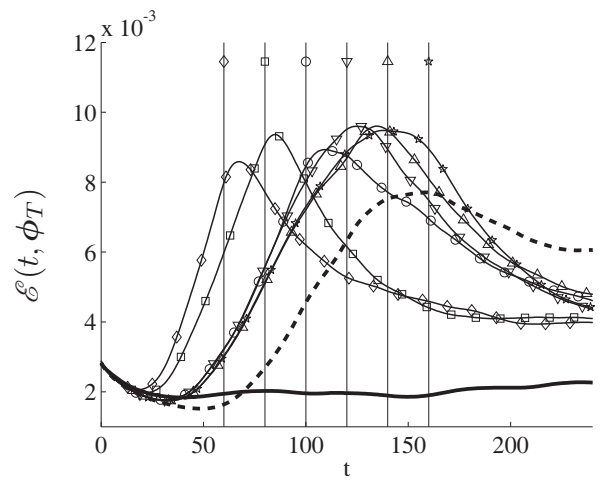

FIG. 11. Evolution of the enstrophy in time for flows optimized toward $\mathcal{J}_{\mathrm{DR}}$ starting from $\phi_{B}$ and $E_{0}=10^{-4}$. Symbols: $T=60(\diamond), T=80(\square), T=100$ $(\bigcirc), T=120(\nabla), T=140(\triangle), T=160(\star)$; also shown is enstrophy evolution of initial condition $\boldsymbol{\phi}_{A}(-)$ and $\boldsymbol{\phi}_{B}(--)$. sition at $t=40$. Results for $t=160$ are not shown here. In this case, the flow is fully turbulent, dominated by small-scale turbulent structures, and the $\lambda_{2}$ structures look visually quite similar to those shown in Fig. 6.

For group A, we do not observe meaningful differences between visualizations of flows optimized toward different time horizons. It is appreciated in Figs. 13(a)-13(d) that the selected plots at $T=80$ and $T=120$ are quite similar, and they are representative for the type of structures observed for

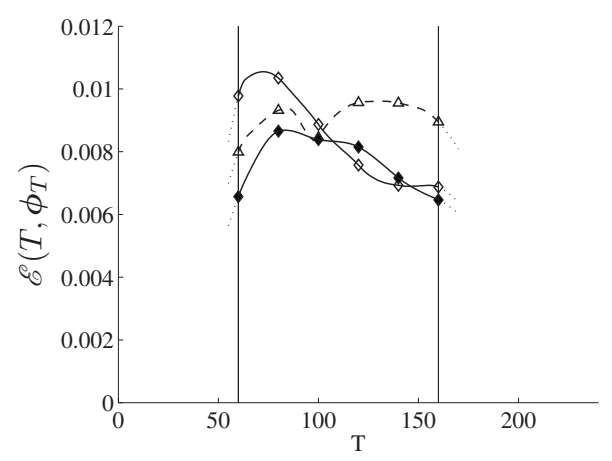

FIG. 12. Maximal enstrophy as a function of time horizon $T$ for flows optimized toward $\mathcal{J}_{\mathrm{DR}} \cdot(-\diamond)$ group $\mathrm{A}, E_{0}=10^{-4} ;(--\triangle)$ group $\mathrm{B}, E$ $=10^{-4}$; and $(-\downarrow)$ group A, $E=10^{-5}$ (lines are obtained by interpolation using splines). 


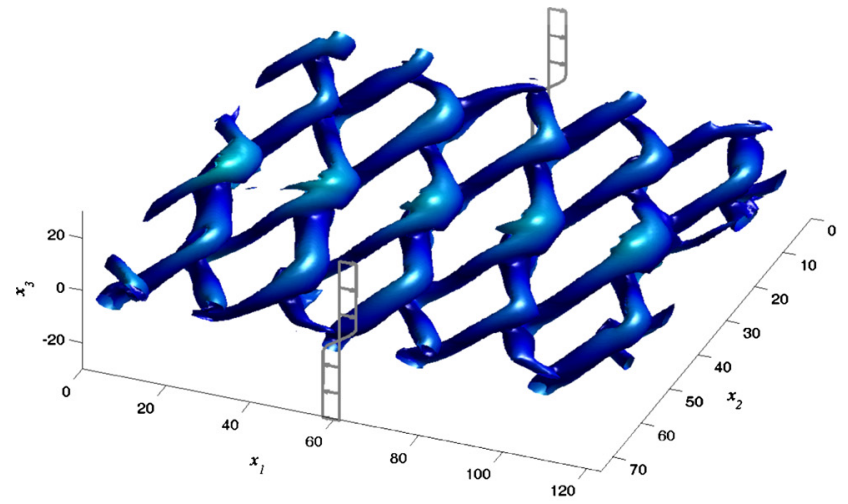

(a) $E_{0}=10^{-5}$, group A, $T=80$

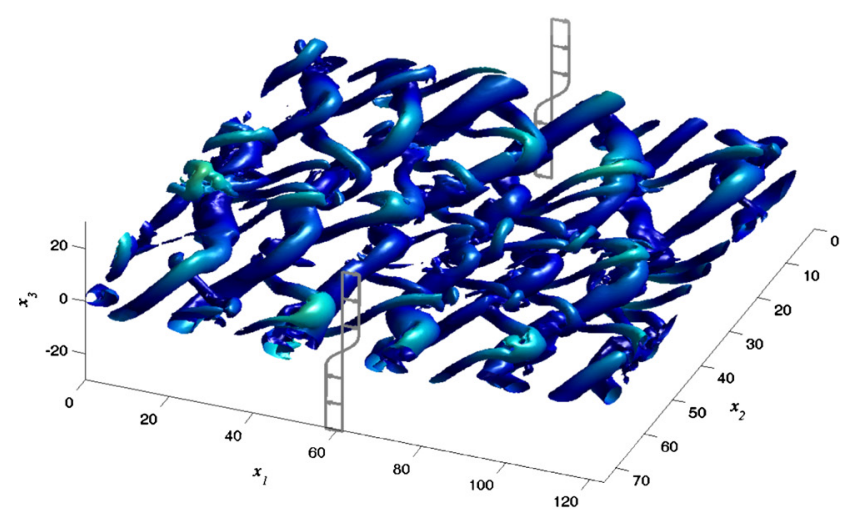

(c) $E_{0}=10^{-4}$, group A, $T=80$

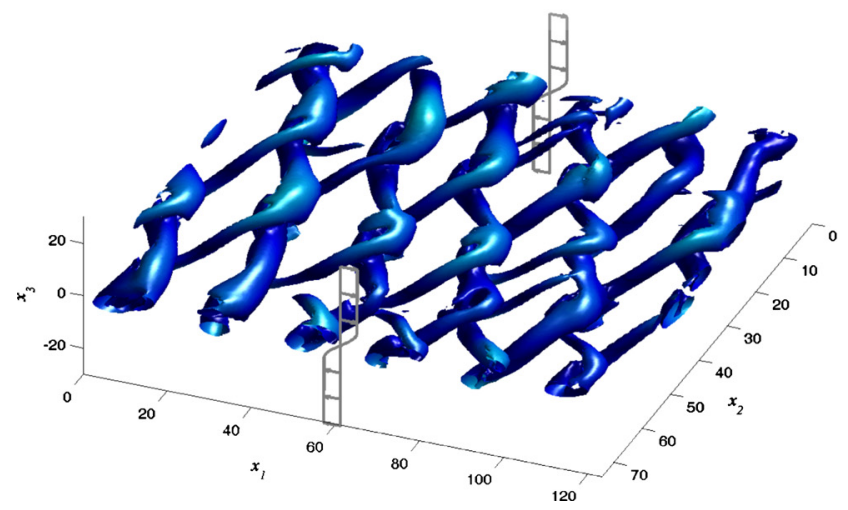

(e) $E_{0}=10^{-4}$, group B, $T=80$

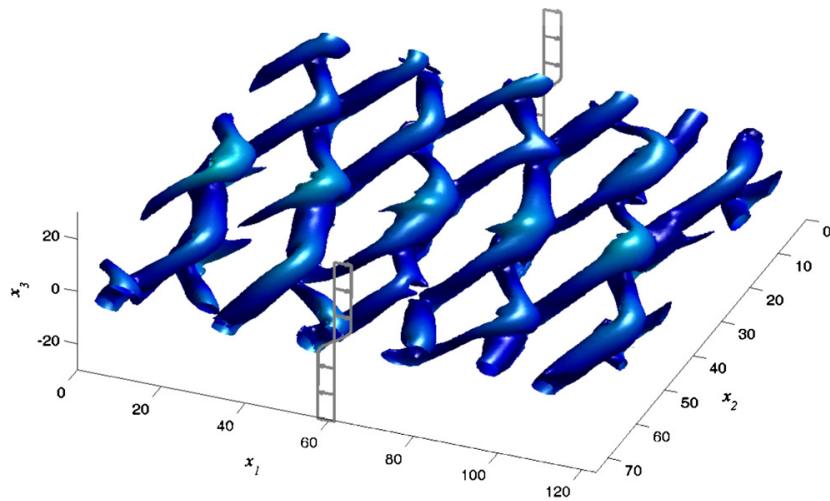

(b) $E_{0}=10^{-5}$, group A, $T=120$

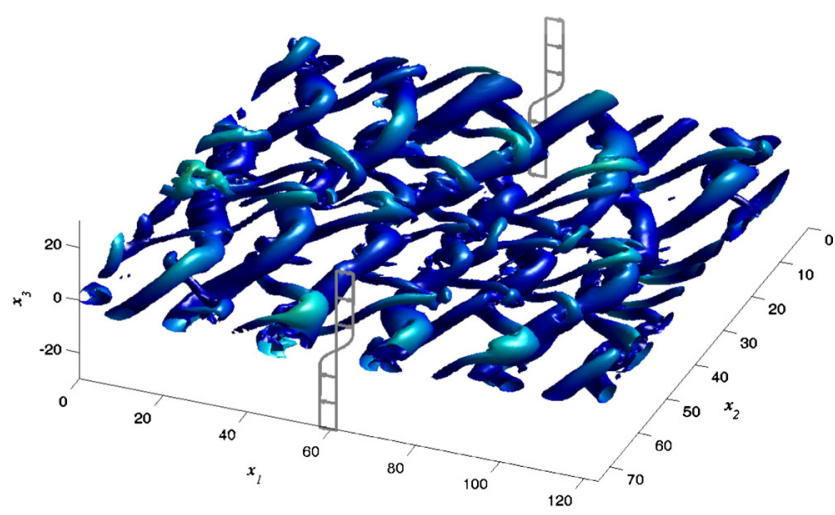

(d) $E_{0}=10^{-4}$, group A, $T=120$

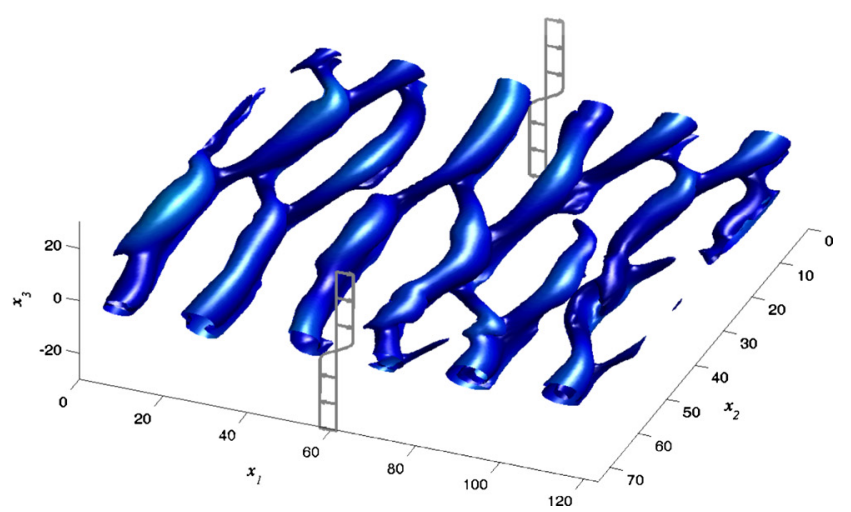

(f) $E_{0}=10^{-4}$, group B, $T=120$

FIG. 13. (Color online) $\lambda_{2}$ visualization of the velocity field for parameters optimized toward $\mathcal{J}_{\mathrm{DR}}$ (colored online with the distance from the mixing center plane); profile $\left\langle u_{1}\right\rangle$ (一); all visualizations at $t=40$.

other time horizons. For group B, more pronounced differences are visible when the time horizon is changed. The flow structures for group B can be divided into two groups according to their time horizon: $T \leq 80$ and $T \geq 100$. The typical structures for $T \leq 80$ are presented in Fig. 13(e) using the case $T=80$. At $t=40$, structures show a diamond-shape pattern of vorticity in the mixing plane. For $T \geq 100$, typical structures are shown in Fig. 13(f) (using $T=120$ ). The vorticity is now more two-dimensional, mainly distributed in span-wise vorticity. In this case, the initial energy is more 


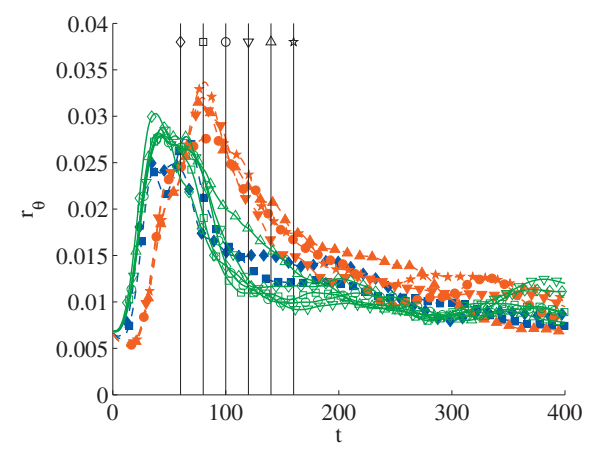

(a) $r_{\theta}$

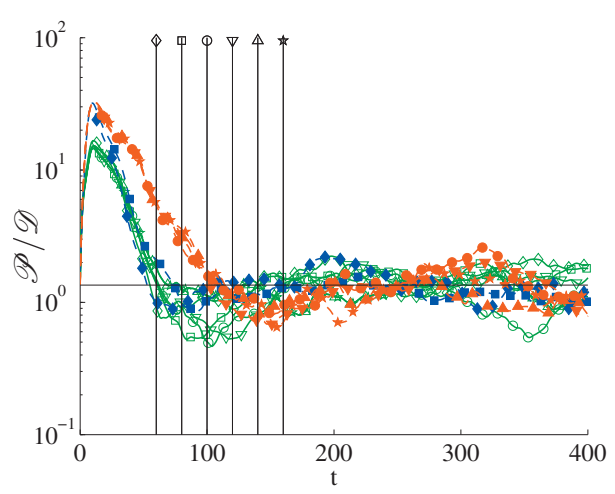

(b) $\mathscr{P} / \mathscr{D}$

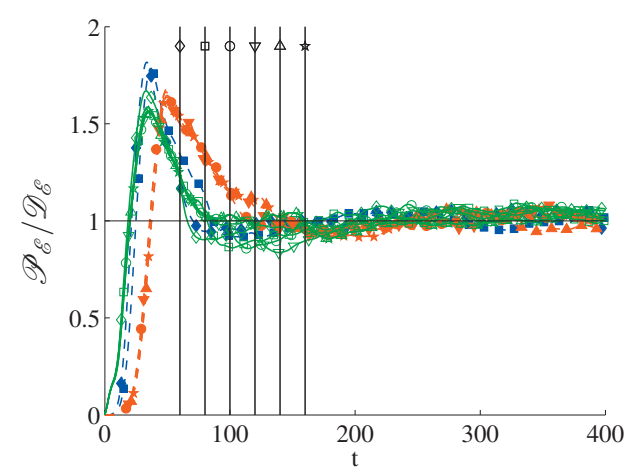

(c) $\mathscr{P}_{\mathscr{E}} / \mathscr{D}_{\mathscr{E}}$

FIG. 14. (Color) (a) Evolution of the momentum thickness growth rate $r_{\theta}$. (b) Evolution of $\mathcal{P} / \mathcal{D}$ in the center of the layer in time. Horizontal line at 1.35. (c) Evolution of the rate of enstrophy production to enstrophy dissipation in time. Horizontal line at 1.0. [(a)-(c)] Flows optimized toward $\mathcal{J}_{\mathrm{DR}}$ with $T=60(\diamond)$, $T=80(\square), T=100(\bigcirc), T=120(\nabla), T=140(\triangle), T=160(\star)$, A-set (—, empty green symbols), and B-set ( -- , filled symbols, $T \leq 80$ blue, $T \geq 100$ red), $E_{0}=10^{-4}$.

stored in two-dimensional structures, such that vortex stretching, break-up of vortices, and subsequent dissipation are "postponed" to later times compared to the flows evolving from Figs. 13(a)-13(e), such that also the enstrophy rise (e.g., observed in Fig. 11) is significantly shifted to larger time horizons.

\section{B. Discussion}

We now turn back to the discussion on control of dissipation and effects on the ensuing self-similar region. By combining the best optima from group A and group B in Sec. IV A, we found that the dissipation rate may be controlled up to the time horizon with an enstrophy level at the time horizon which is almost three times higher than the level of enstrophy observed in the self-similar region of case 1 .

To evaluate self-similarity, the time evolution of the momentum thickness, the ratios $\mathcal{P} / \mathcal{D}$ and $\mathcal{P}_{\mathcal{E}} / \mathcal{D}_{\mathcal{E}}$ [cf. the definitions in Eqs. (19)-(22)] are displayed in Fig. 14 for all optimal solutions from case 2 with control energy $E_{0}=10^{-4}$. We first focus on the peaks in Figs. 14(a)-14(c). It is appreciated that the optimal solutions from case 2 roughly fall apart into two sets. The first set consists of all optima from group A and optima from group B with $T \leq 80$. The second set contains optima from group B with $T \geq 100$. For the momentum thickness growth rate [Fig. 14(a)] and the enstrophy growth rate [Fig. 14(b)], the first set has maxima earlier in time, respectively, at $t \approx 50$ and $t \approx 33$, compared to $t \approx 80$ and $t \approx 50$ for the second set (see also Table I). For the ratio $\mathcal{P} / \mathcal{D}$ in Fig. 9(c), peaks are at the same location in time, but have a different width. This partition into two sets also corresponds with the difference in coherent structures (more three-dimensional versus more two-dimensional) observed at the end of Sec. IV A for all these cases.

These results for case 2 are now compared to results for optimization toward maximum dissipated energy [case 1, cf. Figs. 8, 9(a), and 9(b)]. Peaks in $r_{\theta}, \mathcal{P} / \mathcal{D}$, and $\mathcal{P}_{\mathcal{E}} / \mathcal{D}_{\mathcal{E}}$ are listed in Table I. It is appreciated for case 2 that peaks shift to later times compared to case 1. Clearly, the high dissipation rates at the time horizon dictated by the cost functional used for case 2 are realized by the optimal controls via a shift in

TABLE I. Location in time of the peak in momentum thickness growth rate $r_{\theta}$, rate of production to dissipation of turbulent kinetic energy $\mathcal{P} / \mathcal{D}$, and rate of production to dissipation of enstrophy $\mathcal{P}_{\mathcal{E}} / \mathcal{D}_{\mathcal{E}}$ evaluated for different optima.

\begin{tabular}{lccc}
\hline \hline & $r_{\theta}$ & $\mathcal{P} / \mathcal{D}$ & $\mathcal{P}_{\mathcal{E}} / \mathcal{D}_{\mathcal{E}}$ \\
\hline Case 1 $\left(\mathcal{J}_{\mathrm{DE}}\right)$ & 30 & 8 & 25 \\
Case 2 $\left(\mathcal{J}_{\mathrm{DR}}\right)$ group A, group B with $T \leq 80$ & 50 & 11 & 33 \\
Case 2 $\left(\mathcal{J}_{\mathrm{DR}}\right)$ group B with $T \geq 100$ & 80 & 11 & 50 \\
\hline \hline
\end{tabular}




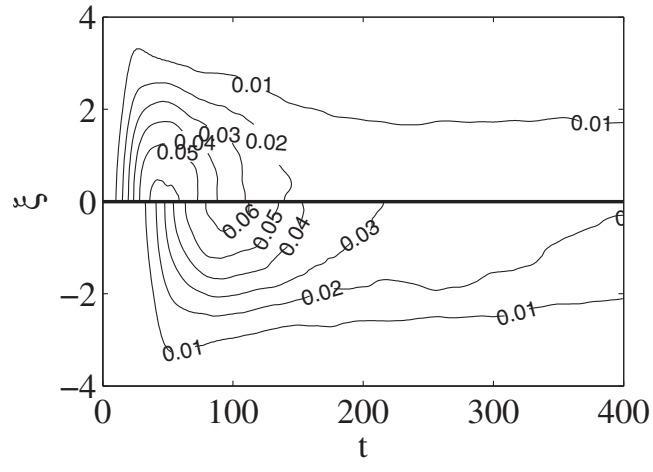

(a) $T=140$

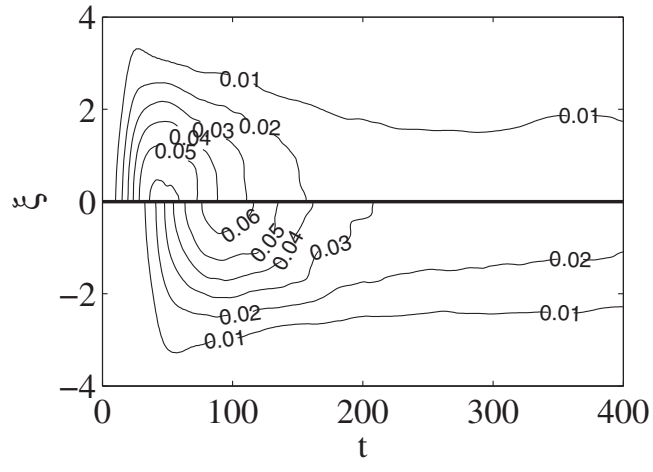

(b) $T=160$

FIG. 15. Evolution in time of the profile $0.5\left\langle u^{\prime} u^{\prime}+v^{\prime} v^{\prime}+w^{\prime} w^{\prime}\right\rangle / \Delta U^{2}$ in function of the scaled normal coordinate $\xi=x_{3} / \theta$. Flows optimized to $\mathcal{J}_{\mathrm{DE}}$ (upper part figure) and $\mathcal{J}_{\mathrm{DR}}$ (lower part figure) for $T=140$ (left) and $T=160$ (right). Energy of the controls $E_{0}=10^{-4}$.

time of the peak in enstrophy growth rate. These shifts also have a large impact on the onset of self-similarity.

Figure 14(a) shows that all optima in case 2 are still evolving, and even up to $t=400$ do not reach self-similarity. Hence, the onset of self-similarity for case 2 starts significantly later than observed in Sec. III B for case 1. To further illustrate this, normalized profiles of turbulent kinetic energy are shown in Fig. 15 for $\mathcal{J}_{\mathrm{DE}}$ and $\mathcal{J}_{\mathrm{DR}}$ (group B) at $T=140$ and 160. The figure shows that the profiles of case $1\left(\mathcal{J}_{\mathrm{DE}}\right)$ become self-similar from $t \approx 160-200$ onward; the normalized turbulent kinetic-energy profiles for case $2\left(\mathcal{J}_{\mathrm{DR}}\right)$ are still changing up to $t=400$. Thus, optimization in case 2 shows that dissipation rates may be increased considerably via dedicated optimal controls, but as a result the transition to self-similarity shifts further back in time.

\section{CONCLUSION}

In the current work, the optimal control of dissipation in a turbulent mixing layer is elaborated. Controls correspond to a set of perturbations on a tangent-hyperbolic mean velocity profile used to initialize the mixing layer in a temporal framework. A suite of DNS-based optimizations is performed with different optimization time windows ( $T$ $=60,80, \ldots, 160)$, including two different energy levels for the controls $E_{0}=10^{-4}$ and $E_{0}=10^{-5}$, and studying two different cost functionals which are relevant for the dissipation of kinetic energy in a shear layer. Also, the effect of optimal controls (aiming to influence dissipation) on the transition of the mixing layer toward a self-similar state is investigated.

In a first case (case 1), optimal control is used to maximize the total dissipated energy in the mixing layer. It is observed that the optimal control for the largest time window $(T=160)$ is optimal for any of the time windows $(60 \leq T$ $\leq 160)$ considered in this study. This suggests that, for sufficiently large time windows in the transition zone, optimal controls are found to become independent of the time window (i.e., by using the controls found at the largest time horizon for all lower time horizons). Results further indicate that these controls facilitate a fast transition of the mixing layer to self-similarity. The coherent turbulent structures during early transition display a diamond-shaped pattern similar to, e.g., patterns observed in experiments in a forced mixing layer by Nygaard and Glezer. ${ }^{36}$ We further found that an approximation of the optimal control with only a few modes (i.e., 4-8) leads to a control which realizes $75 \%$ of the optimal dissipated kinetic energy.

We evaluated (for case 1) the mixing-layer evolution for simulation times beyond the optimization time horizon, and found that optimization of the dissipated energy fastens up the evolution to a self-similar state. The onset of selfsimilarity is observed at a Reynolds number which is a factor of 4-5 lower than observed in the mixing-layer studies by Rogers and Moser ${ }^{3}$ and Balaras et al. ${ }^{4}$ The level of total enstrophy observed in our work is the same, and the ratio of production and dissipation of turbulent kinetic energy in our optimized cases (i.e., $\mathcal{P} / \mathcal{D} \approx 1.35$ ) also corresponded well with values reported in Rogers and Moser. ${ }^{3}$

Since optimal controls for maximization of dissipated energy become independent of the time horizon, and since these controls lead to a fast transition to self-similarity where the dissipation levels are constant (and also at the same level as observed in uncontrolled mixing layers), we surmise that control actions which optimize total dissipated energy do not influence the self-similar region. This also implies that for $T \rightarrow \infty$ this type of controls may become ineffective. To further investigate this, we considered a second case (case 2) in which the dissipation rate at the time horizon is maximized.

For case 2, we observed that it was possible to increase the dissipation rate at the time horizon up to levels which are almost three times higher than the self-similar level of dissipation. We found two different branches of optima depending on the initial guess used to start up the optimization procedure. The first group (A) of optimal solutions is best for smaller time horizons $T \leq 100$ but yields inferior optima for $T>100$. Optima from the second group (B) are best for $T$ $>100$. The difference between optima good for $T \leq 100$ and optima for $T>100$ was identified by looking at coherent structures during the initial transition of the mixing layer. For large time horizons $(T \geq 100)$ it is observed that vorticity is initially more allocated in two-dimensional modes, postponing the development of enstrophy toward the higher optimization time horizons. 
Focusing for case 2 on group B and $T=160$ (after which self-similarity emerges in the corresponding case 1 optimum), we also observe an enstrophy level at the time horizon which is significantly higher than the self-similar level. In comparison to optima from case 1 (optimizing total dissipated energy), this leads to a shift to later times-in our simulation with more than 200 nondimensional time units - of the onset of self-similarity. In the self-similar region, the same levels of enstrophy, etc., are detected as in case 1 .

\section{ACKNOWLEDGMENTS}

S.D. is supported by a scholarship of the Institute for the Promotion of Innovation through Science and Technology in Flanders (IWT-Vlaanderen). The research of J.M. is supported by the Optimization in Engineering CenterK.U.Leuven (OPTEC) and by the Research FoundationFlanders (FWO-Vlaanderen). All simulations were performed on the HPC-cluster VIC3 of K.U.Leuven.

${ }^{1}$ I. Wygnansk and H. E. Fiedler. "The 2-dimensional mixing region," J. Fluid Mech. 41, 327 (1970).

${ }^{2}$ R. G. Batt, "Turbulent mixing of passive and chemically reacting species in a low-speed shear-layer," J. Fluid Mech. 82, 53 (1977).

${ }^{3}$ M. M. Rogers and R. D. Moser "Direct simulation of a self-similar turbulent mixing layer," Phys. Fluids 6, 903 (1994).

${ }^{4}$ E. Balaras, U. Piomelli, and J. M. Wallace, "Self-similar states in turbulent mixing layers," J. Fluid Mech. 446, 1 (2001).

${ }^{5}$ T. R. Bewley, P. Moin, and R. Temam, "DNS-based predictive control of turbulence: An optimal benchmark for feedback algorithms," J. Fluid Mech. 447, 179 (2001).

${ }^{6}$ D. J. Mavriplis, "Discrete adjoint-based approach for optimization problems on three-dimensional unstructured meshes," AIAA J. 45, 740 (2007).

${ }^{7}$ Z. J. Li, I. M. Navon, M. Y. Hussaini, and F. X. Le Dimet, "Optimal control of cylinder wakes via suction and blowing," Comput. Fluids 32, 149 (2003).

${ }^{8}$ M. J. Wei and J. B. Freund, "A noise-controlled free shear flow," J. Fluid Mech. 546, 123 (2006).

${ }^{9}$ G. L. Brown and A. Roshko, "Density effects and large structure in turbulent mixing layers," J. Fluid Mech. 64, 775 (1974).

${ }^{10}$ P. Comte, M. Lesieur, and E. Lamballais, "Large-scale and small-scale stirring of vorticity and a passive scalar in a 3-D temporal mixing layer," Phys. Fluids A 4, 2761 (1992).

${ }^{11}$ M. M. Rogers and R. D. Moser, "The 3-dimensional evolution of a plane mixing layer-The Kelvin-Helmholtz rollup," J. Fluid Mech. 243, 183 (1992).

${ }^{12}$ R. D. Moser and M. M. Rogers, "The 3-dimensional evolution of a plane mixing layer-Pairing and transition to turbulence," J. Fluid Mech. 247, 275 (1993).

${ }^{13}$ B. Vreman, B. Geurts, and H. Kuerten, "Large-eddy simulation of the turbulent mixing layer," J. Fluid Mech. 339, 357 (1997).

${ }^{14} \mathrm{~A}$. Michalke, "On the inviscid instability of the hyperbolic-tangent velocity profile," J. Fluid Mech. 19, 543 (1964).

${ }^{15}$ P. A. Monkewitz and P. Huerre, "Influence of the velocity ratio on the spatial instability of mixing layers," Phys. Fluids 25, 1137 (1982).
${ }^{16}$ R. T. Pierrehumbert and S. E. Widnall, "The two-dimensional and 3-dimensional instabilities of a spatially periodic shear-layer," J. Fluid Mech. 114, 59 (1982).

${ }^{17}$ C. P. Caulfield and W. R. Peltier, "The anatomy of the mixing transition in homogeneous and stratified free shear layers," J. Fluid Mech. 413, 1 (2000).

${ }^{18}$ P. J. Schmid and D. S. Henningson, Stability and Transition in Shear Flows, Applied Mathematical Science Vol. 142 (Springer-Verlag, Berlin, 2001).

${ }^{19}$ P. J. Schmid, "Nonmodal stability theory," Annu. Rev. Fluid Mech. 39, 129 (2007)

${ }^{20}$ S. Delport, M. Baelmans, and J. Meyers, "Constrained optimization of turbulent mixing-layer evolution," J. Turbul. 10, 18 (2009).

${ }^{21}$ W. H. Press, S. A. Teukolsky, W. T. Vetterling, and B. P. Flannery, Numerical Recipes in FORTRAN 77: The Art of Scientific Computing, 2nd ed. (Cambridge University Press, Cambridge, 1996).

${ }^{22}$ D. G. Luenberger, Linear and Nonlinear Programming, 2nd ed. (Kluwer, Boston, 2003).

${ }^{23}$ J. Nocedal and S. J. Wright, Numerical Optimization, 2nd ed. (Springer, New York, 2006).

${ }^{24}$ R. P. Brent, Algorithms for Minimization Without Derivatives, Automatic Computation (Prentice-Hall, Englewood Cliffs, NJ, 1973).

${ }^{25}$ D. G. Luenberger, Optimization by Vector Space Methods (Wiley, New York, 1969).

${ }^{26}$ O. Pironneau, "On optimum design in fluid mechanics," J. Fluid Mech. 64, 97 (1974).

${ }^{27}$ A. Jameson, “Aerodynamic design via control theory,” J. Sci. Comput. 3, 233 (1988).

${ }^{28}$ A. Jameson, L. Martinelli, and N. A. Pierce, "Optimum aerodynamic design using the Navier-Stokes equations," Theor. Comput. Fluid Dyn. 10, 213 (1998)

${ }^{29}$ M. B. Giles and N. A. Pierce, "Adjoint equations in CFD: Duality, boundary conditions and solution behavior," AIAA Paper No. 97-1850, 1997.

${ }^{30}$ J. Meyers and P. Sagaut, "Evaluation of Smagorinsky variants in largeeddy simulations of wall-resolved plane channel flows," Phys. Fluids 19, 095105 (2007).

${ }^{31}$ Spectral Methods, Fundamentals in Single Domains, Scientific Computation, edited by C. Canuto, M. Y. Hussaini, A. Quarteroni, and T. A. Zang (Springer, Berlin, 2006).

${ }^{32}$ R. W. C. P. Verstappen and A. E. P. Veldman, "Symmetry-preserving discretization of turbulent flow," J. Comput. Phys. 187, 343 (2003).

${ }^{33}$ M. Hinze, R. Pinnau, M. Ulbrich, and S. Ulbrich, Optimization with PDE Constraints (Springer, Berlin, 2009).

${ }^{34}$ B. Protas, T. R. Bewley, and G. Hagen, "A computational framework for the regularization of adjoint analysis in multiscale PDE systems," J. Comput. Phys. 195, 49 (2004).

${ }^{35}$ J. Jeong and F. Hussain, "On the identification of a vortex," J. Fluid Mech. 285, 69 (1995).

${ }^{36}$ K. J. Nygaard and A. Glezer, "Core instability of the spanwise vortices in a plane mixing layer," Phys. Fluids A 2, 461 (1990).

${ }^{37}$ S. S. Collis, S. K. Lele, R. D. Moser, and M. M. Rogers, "The evolution of a plane mixing layer with spanwise nonuniform forcing," Phys. Fluids 6, 381 (1994).

${ }^{38}$ R. D. Moser, M. M. Rogers, and D. W. Ewing, "Self-similarity of timeevolving plane wakes," J. Fluid Mech. 367, 255 (1998).

${ }^{39}$ S. B. Pope, Turbulent Flows, 1st ed. (Cambridge University Press, Cambridge, 2000).

${ }^{40} \mathrm{~A}$ procedure similar to the one discussed in Sec. II B is used, but using optimization to $T=140$ as first point in the series. 